\title{
Comparison of static and mineralogical ARD prediction methods in the Nordic environment
}

\author{
Teemu Karlsson (1) - Marja Liisa Räisänen • \\ Marja Lehtonen • Lena Alakangas
}

Received: 13 February 2018 / Accepted: 30 October 2018 / Published online: 13 November 2018

(C) The Author(s) 2018

\begin{abstract}
Acid rock drainage (ARD) is a major problem related to the management of mining wastes, especially concerning deposits containing sulphide minerals. Commonly used tests for ARD prediction include acidbase accounting (ABA) tests and the net acid generation (NAG) test. Since drainage quality largely depends on the ratio and quality of acid-producing and neutralising minerals, mineralogical calculations could also be used for ARD prediction. In this study, several Finnish waste rock sites were investigated and the performance of different static ARD test methods was evaluated and compared. At the target mine sites, pyrrhotite was the main mineral contributing to acid production (AP). Silicate minerals were the main contributors to the neutralisation potential (NP) at $60 \%$ of the investigated mine sites. Since silicate minerals appear to have a significant role in ARD generation at Finnish mine waste sites, the behaviour of these minerals should be more thoroughly investigated, especially in relation to
\end{abstract}

\footnotetext{
T. Karlsson $(\bowtie) \cdot$ M. L. Räisänen Industrial Environments and Recycling Unit, Geological Survey of Finland, P.O. Box 1237, 70211 Kuopio, Finland

e-mail: teemu.karlsson@gtk.fi

T. Karlsson · L. Alakangas

Department of Civil, Environmental and Natural Resources Engineering, Division of Geosciences and Environmental Engineering, Luleå University of Technology, 97187 Luleå, Sweden

M. Lehtonen

Mineral Processing and Materials Research Unit, Geological Survey of Finland, P.O. Box 96, 02151 Espoo, Finland
}

the acid produced by pyrrhotite oxidation. In general, the NP of silicate minerals appears to be underestimated by laboratory measurements. For example, in the NAG test, the slower-reacting NP-contributing minerals might require a longer time to react than is specified in the currently used method. The results suggest that ARD prediction based on SEM mineralogical calculations is at least as accurate as the commonly used static laboratory methods.

Keywords ABA test - NAG test - SEM - Waste rock · Risk assessment

\section{Introduction}

Acid rock drainage (ARD) with high concentrations of potentially harmful elements is considered as one of the main concerns related to the management of mining wastes (e.g. MEND 1991; Price 2003, Dold 2014; Mehta et al. 2018). This particularly applies to deposits containing sulphide minerals, which are prone to oxidisation under the influence of atmospheric conditions (e.g. Singer and Stumm 1970; Blowes and Ptacek 1994). Drainage quality largely depends on the mineralogical and chemical composition of the mine wastes, and particularly on the ratio of acid-producing and neutralising minerals (e.g. Blowes and Jambor 1990; Blowes and Ptacek 1994), combined with reactions catalysed by microbes (e.g. Singer and Stumm 1970). The main acid-producing sulphide species include iron sulphides, such as pyrite $\left(\mathrm{FeS}_{2}\right)$ and pyrrhotite $\left(\mathrm{Fe}_{1-x} \mathrm{~S}\right)$, 
whereas the most common neutralising minerals are relatively fast-reacting carbonates (e.g. calcite $\left(\mathrm{CaCO}_{3}\right)$ and dolomite $\left(\mathrm{CaMg}\left(\mathrm{CO}_{3}\right)_{2}\right)$ and slower-reacting silicates, e.g. mica and chlorite (e.g. Ptacek and Blowes 1992; Blowes and Ptacek 1994; Plumlee 1999; Becker et al. 2015). The main sulphide oxidation and acid neutralisation reactions are presented in more detail, for example, by Seal and Hammarstrom (2003) and Dold (2010).

Since ARD plays a major role in the generation of environmental issues, the accurate prediction of ARD is of utmost importance (e.g. Parbhakar-Fox and Lottermoser 2015; Dold 2017). Nevertheless, prediction of the ability of mine waste materials to produce ARD is sometimes challenging, as ARD generation depends on various mineralogical, chemical, hydrological and microbiological factors (e.g. Blowes and Jambor 1990; Nordstrom 2000; Dold 2010; Blowes et al. 2014). The characterisation methods for assessing the acid production potential (APP) and ARD risk can be divided into static and kinetic tests (Lapakko 2002). Static tests are short-term (duration usually measured in hours or days) laboratory analyses. They are relatively low cost (around tens of US\$) and usually used for preliminary investigation and screening. Kinetic tests are long-term tests (from several months to years), revealing information on the time scale of drainage events (Lapakko 2002). Kinetic tests are more expensive (from approximately hundreds to thousands of US\$) and require site-specific planning.

The most commonly used static APP prediction tests are acid-base accounting (ABA) tests, which include determination of the sulphur/sulphide content of rock material to calculate the acid potential (AP) and determination of the neutralisation potential (NP). The potential risk can then be estimated based on the ratio of the NP and AP (neutralisation potential ratio, NPR) or the subtraction NP-AP (net neutralisation potential, NNP), as is customary in Finland, for instance. It is commonly assumed that mine waste is potentially acid-generating if NPR $<1$ or NNP $<-20$, in the uncertainty zone if NPR is $1-3$ or NNP is between -20 and +20 , and non-acid-generating if NPR $>3$ or NNP $>$ 20 (Sobek et al. 1978; White et al. 1999; Price 2009).

Many variations of the ABA test exist, including the standard ABA originally developed for coal mining (Sobek et al. 1978), the modified ABA (Lawrence et al. 1989; Lawrence and Wang 1997) and "acid addition on the basis of the carbonate content" (EN 15875), which is a recommended standard method within
Europe. The Finnish mine waste decree 190/2013 (Finnish Government Decree 2013), which is based on European legislation (EC 2009), states that mine waste can be considered inert if the sulphidic $\mathrm{S}$ concentration is $\leq 0.1 \%$ or the sulphidic S concentration is $\leq 1 \%$ and NPR determined by the standard method EN 15875 is > 3 , given that the other requirements mentioned in the decree are also fulfilled. The AP is usually calculated based on the total sulphur content of the sample and expressed as a pyrite equivalent, since it is assumed that pyrite is the most common sulphide mineral and that $4 \mathrm{~mol}$ of protons is produced during the oxidation process (Colmer and Hinkle 1947; Singer and Stumm 1970; Nordstrom 2000; Dold 2014). The NP is typically estimated by titrating the sample with acid (Price et al. 1997; White et al. 1999), but it can also be calculated, for example, based on the carbonate carbon content of the rock material (Lawrence and Wang 1997).

The ABA method has some disadvantages. Some carbonate minerals containing iron, particularly siderite $\left(\mathrm{FeCO}_{3}\right)$, do not necessarily contribute to neutralisation (Lawrence and Wang 1997; Haney et al. 2006). The method does not take into account the reactive noncarbonate minerals that may contribute to acid neutralisation, e.g. easily dissolving silicate minerals. Moreover, it does not consider the groups of Fe(III) hydroxides and $\mathrm{Fe}(\mathrm{II})$ hydroxide sulphates together with metal chlorides and sulphates as a proton source, although they might be a significant source of acidity (Dold 2017). Furthermore, in the ABA test, the AP is calculated by multiplying the wt\% of $\mathrm{S}$ by the factor 31.25 based on the hypothesis that $2 \mathrm{~mol}$ of protons (released from pyrite oxidation) are neutralised by $1 \mathrm{~mol}$ of calcite (e.g. Gard Guide; Verburg et al. 2009). However, at a circumneutral $\mathrm{pH}$, the most common carbonate species is bicarbonate (Appelo and Postma 2010), and the calculation factor should then be 62.5 , as two times more calcite is needed to neutralize the same quantity of protons (Dold 2017).

Another commonly used static test for ARD prediction is the net acid generation (NAG) test, which is based on the reaction of a sample with hydrogen peroxide $\left(\mathrm{H}_{2} \mathrm{O}_{2}\right)$, which accelerates the oxidation of sulphide minerals in the sample. During the test, acid generation and acid neutralisation reactions can occur simultaneously, with the end result representing a direct measurement of the net amount of acid generated by the sample (Miller et al. 1997; Stewart et al. 2006). As the test does not estimate the neutralisation potential (Miller 
and Jeffery 1995), the use of the net acid production potential (NAPP) together with the NAG pH for more a detailed classification of acid generation is recommended (Morin and Hutt 1999; Räisänen et al. 2010). One of the main advantages of the NAG test is that it does not require separate sulphur determinations. The test is also easier to conduct in a field laboratory than other static tests. As a disadvantage, the test does not give a value for the neutralisation potential, and additional NP measurement is therefore required to calculate the NAPP. Some contradictory predictions have also been noted when NAG test results have been compared with ABA results (Morin and Hutt 1999).

The widely used static ABA and NAG tests have known limitations related to the mineralogy of the sample material (White III et al. 1999; Paktunc 1999b; Jambor 2003; Parbhakar-Fox and Lottermoser 2015; Dold 2017; Parbhakar-Fox et al. 2018). For example, the APP may be overestimated if there are other sulphide- or sulfur-containing minerals than rapidly acid-producing pyrite or pyrrhotite. Conversely, the APP may be underestimated if the waste contains large amounts of easily dissolvable and acid-generating iron sulphate minerals or siderite. The NP may be underestimated if the weathering of silicate minerals is not considered in APP estimations (Lawrence and Scheske 1997; Paktunc 1999b). On the other hand, the NP determined in the laboratory using strong acids will sometimes overestimate silicate mineral reactivity and hence the NP (Lawrence and Scheske 1997; Jambor and Blowes 1998). In addition, static tests do not indicate the source minerals of the NP and AP (Lawrence and Scheske 1997; Paktunc 1999b). Therefore, some mineralogical-based approaches have been proposed, e.g. by Lawrence and Scheske (1997), Parbhakar-Fox and Lottermoser (2015) and Jamieson et al. (2015). As pointed out by Dold (2017), scanning electron microscopy (SEM)-based automated mineral quantification methods have considerably developed in the last decades, increasing the available mineralogical data that could be utilised in ARD prediction.

In this study, we compared the ABA test as presented in the standard EN 15875, the single-addition NAG test as presented in the AMIRA guidebook (Smart et al. 2002), SEM mineralogy-based ARD predictions based on Lawrence and Scheske (1997) and Dold (2017) and actual drainage quality at the target mine sites. For the ABA test, the NP was determined by standard titration and was also calculated based on the carbonate content, as presented by Lawrence and Wang (1997). The AP was calculated with the commonly used factor of 31.25 and the factor of 61.5 proposed by Dold (2017), and the results were compared. Data and samples from several Finnish mine waste sites were utilised, representing a wide range of deposit types.

The objective of this study was to assess the functionality of standard static and mineralogical ARD prediction methods in the Nordic environment and whether static laboratory ARD tests could be substituted in some cases by an ARD prediction based only on SEM mineralogy. Furthermore, the ARD-related properties of various Finnish waste rock sites, including the main minerals responsible for neutralisation and the acid generation potential, are presented.

\section{Materials and methods}

In total, 14 waste rock and 14 corresponding seepage water samples were collected from 10 Finnish mine sites representing various Archaean and Palaeoproterozoic deposits, waste rock types and waste rock facilities of different ages (Table 1). The sampling was conducted during various fieldwork campaigns during the years 2014-2017. The waste rock samples consisted of around $15-20 \mathrm{~kg}$ of heterogeneous rock material randomly collected from the surface of the waste rock piles above the seepage points. From the Hitura target site, two sets of waste rock and water samples were taken from the same place: the first in 2014 and the second in 2016. The Kylylahti mine site was first visited for waste rock and water samples in 2014 and thereafter revisited for an additional water and rock sample from the same location in 2017. For this second Kylylahti rock sample, which was clearly more weathered than during the first sampling, only static laboratory tests were conducted, not SEM mineralogy. The Horsmanaho and Siilinjärvi mine sites both had two different waste rock piles, an inactive older one and a new, currently active one, both of which were separately sampled.

The quality assessment of the mine waste drainage included the analysis of $\mathrm{pH}$ and alkalinity, which were measured from small seepage streams flowing from the base of the waste rock piles. In two cases (Horsmanaho new pile and Hammaslahti), no visible flowing stream could be found, and the samples were taken from a pond at the waste rock pile. The measurement was conducted at the site using a portable multi-parameter YSI sonde, 


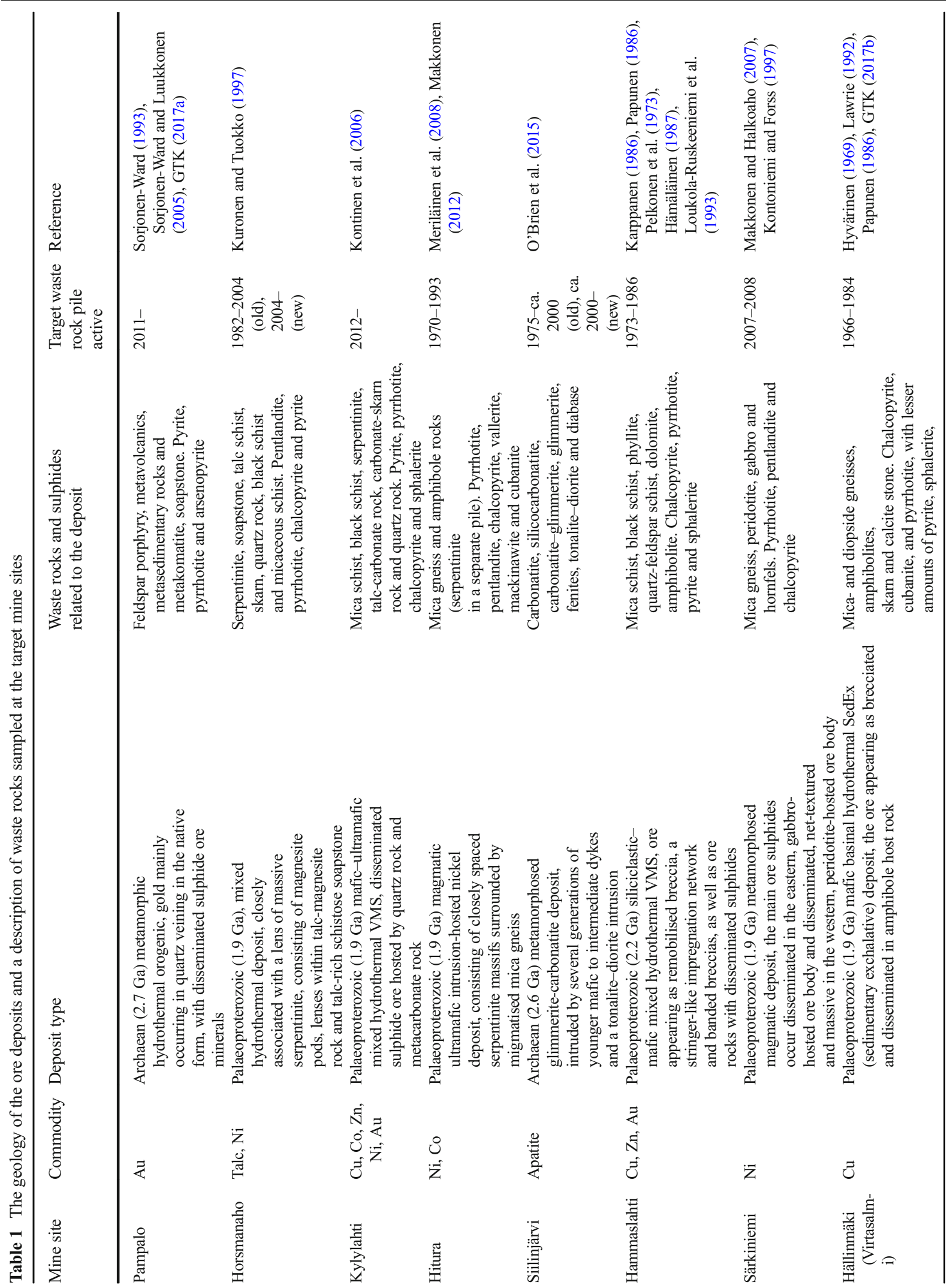


which was calibrated to $\mathrm{pH} 4$ and 7 prior to every field trip. The alkalinity was determined with a Hach titrator with 0.16 or $1.60 \mathrm{~N} \mathrm{H}_{2} \mathrm{SO}_{4}$ to an end point of $\mathrm{pH} 4.5$. The titration $\mathrm{pH}$ was measured with the Mettler Toledo SevenGo $\mathrm{pH}$ meter, which was calibrated daily to $\mathrm{pH} 4$ and 7.

The mine waste samples were analysed in an accredited laboratory (Labtium Oy). Laboratory duplicates for four waste rock samples and certified reference materials were employed for quality control of the analyses. The rock samples were first dried at $<40{ }^{\circ} \mathrm{C}$ and then crushed $(>70 \%,<2 \mathrm{~mm})$. For the different chemical and mineralogical analyses, the crushed samples were split with riffles and/or the cone and quartering method and ground in a steel container. The total concentrations of $\mathrm{C}$ and $\mathrm{S}$ were determined using a pyrolytic method (the Leco method) and IR detection. The amount of carbonate $\mathrm{C}$ was calculated based on the difference between total $\mathrm{C}$ and non-carbonate $\mathrm{C}$. Total $\mathrm{C}$ was determined by pyrolysis and IR detection and non-carbonate $\mathrm{C}$ after hydrochloric acid treatment by pyrolysis and IR detection.

\section{Mineralogical analyses}

Mineralogical analyses were conducted at the GTK Research Laboratory by FE-SEM-EDS: JEOL JSM 7100F Schottky, combined with an Oxford Instruments EDS spectrometer X-Max $80 \mathrm{~mm}^{2}$ (SDD). The crushed and ground sample materials for geochemical analysis were also used to prepare mineralogical polished samples cast in epoxy and covered with graphite to enhance electric conductivity. To investigate the modal mineralogy, around 10,000 individual mineral particles were analysed from each sample utilising Feature software. The results are semi-quantitative and were normalised to $100 \%$.

Mineral identification was based on comparing the numerical element composition obtained from EDS spectra with the mineralogical database of GTK. For technical reasons, usually around $5-10 \%$ of mineral identifications are classed as "unclassified". This class mainly includes mixed analyses generated from various mineral phases. The amount of unclassified minerals is usually larger when analysing fine particle samples and/ or samples with complex mineralogy. Precise identification of minerals based on EDS spectra is not always possible, for instance because carbon and $\mathrm{OH}$ and $\mathrm{H}_{2} \mathrm{O}$ 
groups are not shown in the analysis, which should be taken into account when examining the results.

As a quality control measure for the mineralogical analyses by FE-SEM-EDS, the samples were also analysed by X-ray diffraction (Bruker D8 Discover A25) in order to verify the identification of the main mineral phases. The accuracy of mineral classification based on the GTK mineral database was routinely monitored for each analysed sample. The analytical data that were left unclassified were checked manually.

\section{NAG test}

A single-addition NAG test was performed as proposed by Smart et al. (2002). A mixture of $2.5 \mathrm{~g}$ of pulverized sample and $250 \mathrm{ml}$ of $15 \%(\mathrm{~m} / \mathrm{V}) \mathrm{H}_{2} \mathrm{O}_{2}$ was allowed to react for about $12 \mathrm{~h}$, after which the mixture was boiled until the visible reaction ceased. After cooling, $\mathrm{pH}$ was measured and the suspension was titrated with $\mathrm{NaOH}$ $(0.1 \mathrm{~mol} / \mathrm{L})$ to $\mathrm{pH} 4.5$ and 7.0. The NAG values were calculated based on the amount of $\mathrm{NaOH}$ consumed in the titration and expressed as $\mathrm{kg} \mathrm{H}_{2} \mathrm{SO}_{4} / \mathrm{t}$ (Miller et al. 1997; Smart et al. 2002).

\section{Determination of AP}

The laboratory tests to determine the acid-generating potential of a waste rock sample included the modified ABA test based on the EN 15875 standard (European Committee for standardization 2008). In the ABA test, the AP was calculated by multiplying the wt $\%$ of $\mathrm{S}$ by the factor 31.25 , as proposed in the standard, and by the factor of 62.5 proposed by Dold (2017). The mineralogical AP (minAP) was calculated by multiplying the $\mathrm{wt} \%$ of $\mathrm{S}$ in the mineral with the wt $\%$ of the mineral in the sample to obtain the wt $\%$ of $\mathrm{S}$ in the sample, which was multiplied by 31.25 (EN 15875) or 62.5 (Dold 2017). These factors assume that the oxidation of $1 \mathrm{~mol}$ of pyrite via oxygen produces $4 \mathrm{~mol}$ of $\mathrm{H}^{+}$, and all of the $\mathrm{S}$ is assumed to be generated by pyrite, although in mineralogical AP calculations, the amount of $\mathrm{H}^{+}$produced by different sulphide species is also taken into account; chalcopyrite $\left(\mathrm{CuFeS}_{2}\right)$ and pyrrhotite oxidation produce only $2 \mathrm{~mol}$ of $\mathrm{H}^{+}$, and the factor is therefore divided by 2 , while sphalerite $((\mathrm{Zn}, \mathrm{Fe}) \mathrm{S})$ and galena $(\mathrm{PbS})$ oxidation produce $1 \mathrm{~mol}$ of $\mathrm{H}^{2}$, and the factor is therefore 0 (Dold 2017).

\section{Determination of the NP}

The static test to determine the NP by titration was based on the EN 15875 standard (European Committee for standardization 2008), which was originally a modification of the method by Lawrence and Wang (1997). Unlike the modified ABA test by Lawrence and Wang (1997), the standard EN 15875 method does not include the Fizz test to determine the amount of acid needed. Instead, the addition of the acid is based on the total carbon content determined by the EN 13137:2001 standard. A pulverised sample of $2 \mathrm{~g}$ was mixed with demineralised water, hydrochloric acid $(1.0 \mathrm{~mol} / \mathrm{L})$ was added and the mixture was stirred until $\mathrm{pH} 2.0$ 2.5 was reached after $24 \mathrm{~h}$. If the $\mathrm{pH}$ was above 2.5 at $22 \mathrm{~h}$, more $\mathrm{HCl}$ was added to adjust the $\mathrm{pH}$ between 2.0 and 2.5 and preferably as close to 2.0 as possible. After the stabilisation of $\mathrm{pH}$, the solution was titrated with sodium hydroxide $(0.1 \mathrm{~mol} / \mathrm{L})$ to an end point $\mathrm{pH}$ of 8.3 . The volume of $\mathrm{NaOH}$ indicates how much of the $\mathrm{HCl}$ the sample has consumed, therefore reflecting the neutralisation potential, which was calculated as $\mathrm{CaCO}_{3}$ equivalents in kilograms per ton.

The carbonate NP (carbNP) value was calculated on the basis of the carbonate $\mathrm{C}$ concentration, also yielding the value in units of $\mathrm{kg} \mathrm{CaCO}_{3} / \mathrm{t}$. The carbC value was multiplied by a factor of 83.3 , which was calculated as follows:

$$
\begin{aligned}
& 100.06_{(\text {mole weight calcite })} / 12.01_{(\text {mole weight carbon })} \\
& \times 10_{(\mathrm{tCaCO} 3 / 1000 \mathrm{t})} \\
& =83.3
\end{aligned}
$$

Mineralogical NP (minNP) values were determined by calculations based on mineralogy as presented by Dold (2017) and Lawrence and Scheske (1997), respectively. As calcite is considered to be the most important neutralising mineral in the mining environment, it is commonly considered in calculations for the NP and results are expressed as its equivalent (e.g. Dold 2017). The mineralogical NP for a carbonate mineral converted to $\mathrm{X} \mathrm{kg} \mathrm{CaCO}_{3} / \mathrm{t}$ was calculated by dividing the mole weight of carbon by the total mole weight of the carbonate mineral and then multiplying by the mineral concentration (wt\%) with the factor 83.3. For example, the minNP for $2 \mathrm{wt} \%$ of magnesite $\left(\mathrm{MgCO}_{3}\right)$ was calculated as follows: 


$$
\begin{aligned}
& \operatorname{minNP}_{(\text {magnesite })} \\
&= 12.01_{(\text {mole weight carbon } \mathrm{g} / \mathrm{mol})} / 84.31_{(\text {mole weight magnesite } \mathrm{g} / \mathrm{mol})} \\
& \times 2_{(\text {magnesite wt. } \%)} \times 83.3 \\
&= 23.74 \mathrm{kgCaCO}_{3} / \mathrm{t}
\end{aligned}
$$

Mineralogical NP calculations for non-carbonate minerals were performed according to Lawrence and Scheske (1997) by adding together the weighted NP values for each (significant) mineral present in the sample. To simplify the minNP calculations, non-carbonate minerals classified into slow-weathering, very slow-weathering and inert groups were not taken into account, as their NP contribution is insignificant (Sverdrup 1990; Kwong 1993). In addition to carbonate minerals, only the silicate minerals classified as fast- and intermediate-weathering by Sverdrup (1990) with a total mass $\%$ above 1 were included in the calculations. In contrast to the Cross, Iddings, Pirsson and Washington (CIPW) normative mineralogical composition applied by Lawrence and Scheske (1997), which is not well suited to metamorphosed rocks (Paktunc 1999a), mineralogical compositions for the minNP calculations were obtained by SEM-based automated mineral quantification, as described above.

The relative reactivity values applied in this study were obtained from the original references (Sverdrup 1990, Kwong 1993). Sverdrup (1990) stated that the relative reactivity of minerals changes depending on the total percentages of different mineral groups in the sample (Table 2). To simplify the calculations in this study, the relative reactivity of the average mineral class content of $30 \%$ was used, as this is probably close to the average realistic mineral class contents in a typical rock. Calculated minNP values were converted to $\mathrm{kg} \mathrm{CaCO}_{3}$ by using the molecular weight of $\mathrm{CaCO}_{3}$ for the mineral. The selected minerals and their molecular weights used in this study are presented in Table 3.

For example, the NP contribution in $\mathrm{kg} \mathrm{CaCO}_{3}$ equivalent $/ \mathrm{t}$ for $30.0 \mathrm{wt} \%$ of diopside $\left(\mathrm{CaMgSi}_{2} \mathrm{O}_{6}\right)$ in a sample would be calculated as follows:

$$
\begin{aligned}
\operatorname{minNP}_{(\text {diopside })} \\
\quad=(30.0 / 100) \times(1000 \mathrm{~kg} / 1 \text { tonne }) \\
\quad \times\left(100.06_{(\text {moleweight calcite })} / 216.55_{(\text {mole weight diopside })}\right) \\
\quad \times 0.67=92.9 \mathrm{~kg} \mathrm{CaCO} 3 / \mathrm{t}
\end{aligned}
$$

An example of the mineralogical calculation of the NNP and NPR for a hypothetical sample is presented in Table 4.

\section{Results}

Measured seepage water $\mathrm{pHs}$ and alkalinities are presented in Table 5. Modal mineral contents based on EDS spectra are presented in Table 6. Minerals that occur in larger amounts than $1 \%$ are included, as well as important minerals related to the AP and NP (sulphides and carbonates, respectively) in lesser amounts. The samples included various minerals that could not be precisely identified by EDS analysis, e.g. different $\mathrm{Mg}$ silicates such as serpentine $\left((\mathrm{Mg}, \mathrm{Fe})_{3} \mathrm{Si}_{2} \mathrm{O}_{5}(\mathrm{OH})_{4}\right), \mathrm{Mg}$ amphiboles and $\mathrm{Mg}$ pyroxenes. Therefore, the classification of amphiboles and pyroxenes is indicative, and the division between tremolite $\left(\mathrm{Ca}_{2} \mathrm{Mg}_{5} \mathrm{Si}_{8} \mathrm{O}_{22}(\mathrm{OH})_{2}\right)$ and diopside is uncertain. Although FE-SEM-EDS is not suitable for the identification of minerals containing $\mathrm{OH}$ and $\mathrm{H}_{2} \mathrm{O}$ groups, some small amounts $(0.01-0.02 \mathrm{wt} \%)$ of gypsum $\left(\mathrm{CaSO}_{4} \cdot 2 \mathrm{H}_{2} \mathrm{O}\right)$ and silicate-gypsum mixture were detected in several samples.

Geochemical analysis results are presented in Table 7, together with different APs and NPs calculated using various methods. According to the total $\mathrm{S}$ and carbonate $\mathrm{C}$ concentrations, the waste rock samples can be classified into three groups: samples of group I have minor sulphide sulphur, are close to inert ( $\leq$ $0.1 \mathrm{wt} \% \mathrm{~S}$ ) and have high or moderate carbonate $\mathrm{C}$ contents; samples of group II have a relatively high sulphide sulfur content and high to moderate carbonate $\mathrm{C}$ contents; and samples of group III have variable contents of sulphide sulphur and low or no carbonate carbon contents $(\leq 0.1 \mathrm{wt} \%$ carbC). The contributions of different minerals to the total minNP of the samples are presented in Table 8. From the calculated AP and NP values, different NNPs and NPRs were derived with an AP factor of 31.25 (Table 9) and an AP factor of 62.5 (Table 10).

The main results are presented below according to the target mine site, after which some general observations are summarised.

Pampalo

Drainage water from the Pampalo waste rock pile had a neutral $\mathrm{pH}$ (6.7) and high alkalinity $\left(239 \mathrm{mg} / \mathrm{L} \mathrm{CaCO}_{3}\right)$. The main minerals (wt \% $>5)$ were albite $\left(\mathrm{NaAlSi}_{3} \mathrm{O}_{8}\right.$, 28.6 wt. \%), biotite $\left(\mathrm{K}(\mathrm{Mg}, \mathrm{Fe})_{3}\left(\mathrm{AlSi}_{3} \mathrm{O}_{10}\right)(\mathrm{OH}, \mathrm{F})_{2}\right.$, $18.9 \mathrm{wt} \%)$, k-feldspar ( $\mathrm{KAlSi}_{3} \mathrm{O}_{8}, 15.6 \mathrm{wt} \%$ ) and quartz $\left(\mathrm{SiO}_{2}, 11.2 \mathrm{wt} \%\right)$. The carbonate mineral content was relatively high $(4.44 \mathrm{wt} \%)$, the sample containing 
Table 2 Relative reactivity in terms of the acid neutralisation capacity for selected minerals after Sverdrup (1990) and Kwong (1993)

\begin{tabular}{|c|c|c|c|c|c|}
\hline \multirow[t]{2}{*}{ Mineral class } & \multirow[t]{2}{*}{ Typical minerals } & \multicolumn{4}{|c|}{$\begin{array}{l}\text { Relative reactivity } \\
\text { Average mineral class } \\
\text { content }\end{array}$} \\
\hline & & $100 \%$ & $30 \%$ & $3 \%$ & $0.3 \%$ \\
\hline Carbonates & Calcite, dolomite, magnesite, aragonite, brucite & 1 & 1 & 1 & 1 \\
\hline Fast-weathering & $\begin{array}{l}\text { Anorthite, olivine, garnet, diopside, wollastonite, jadeite, nepheline, leucite, } \\
\text { spodumene }\end{array}$ & 0.6 & 0.67 & 0.3 & 0.1 \\
\hline Intermediate-weathering & $\begin{array}{l}\text { Enstatite, augite, hornblende, tremolite, actinolite, biotite, chlorite, serpentine, } \\
\text { talc, epidote, zoisite, hedenbergite, glaucophane, anthophyllite, phlogopite } \\
\text { anthophyllite }\end{array}$ & 0.4 & 0.2 & 0.03 & 0.01 \\
\hline Slow-weathering & Plagioclase (Ab100-Ab30), kaolinite, vermiculite, montmorillonite, gibbsite & 0.02 & 0.013 & 0.002 & - \\
\hline Very slow-weathering & K-feldspar, muscovite & 0.01 & 0.007 & 0.001 & - \\
\hline Inert & Quartz, rutile, zircon & 0.004 & 0.0007 & - & - \\
\hline
\end{tabular}

In this study, the relative reactivity of the average mineral class content of $30 \%$ was used

${ }^{\mathrm{a}}$ In soil, at $\mathrm{pH} 5$

${ }^{\mathrm{b}}$ Phlogopite added to the intermediate-weathering class based on Schweda and Kalinowski (1994)

${ }^{c}$ Anthophyllite added to the intermediate-weathering class based on Chen and Brantley (1998) and Rozalen et al. (2014)

$4.21 \mathrm{wt} \%$ of dolomite, $0.14 \mathrm{wt} \%$ of siderite and $0.09 \mathrm{wt} \%$ of calcite. The total $\mathrm{C}$ concentration was $0.85 \%$ and the carbonate $\mathrm{C}$ concentration $0.82 \%$. The sulphidic mineral content was low (0.04 wt $\%)$, the sample containing $0.03 \mathrm{wt} \%$ of chalcopyrite and $0.01 \mathrm{wt} \%$ of pyrrhotite. The sulphides appeared as single and unweathered, mainly $<10 \mu \mathrm{m}$ grains. No oxidised Fe sulphides were detected. The total S concentration was $0.1 \%$ based on the Leco method and $0.007 \%$ according to calculation based on the sulphide mineral amounts. Based on the $\mathrm{S}$ and $\mathrm{C}$ concentrations, the Pampalo sample was classified into group I.

The NAG pH of the Pampalo sample was 9.2, both NAG values ( $\mathrm{pH} 4.5$ and $\mathrm{pH} 7.0$ ) being $0 \mathrm{~kg} \mathrm{H}_{2} \mathrm{SO}_{4} / \mathrm{t}$. The AP calculated based on the total $\mathrm{S}$ and applying the factor of 31.25 was $3.13 \mathrm{~kg} \mathrm{CaCO}_{3} / \mathrm{t}$ (AP1) and $6.25 \mathrm{~kg}$ $\mathrm{CaCO}_{3} / \mathrm{t}$ applying the factor of 62.5 (AP2). The minAP calculated based on minS was $0.22 \mathrm{~kg} \mathrm{CaCO}_{3} / \mathrm{t}$ with the factor of 31.25 (minAP1) and $0.44 \mathrm{~kg} \mathrm{CaCO}_{3} / \mathrm{t}$ with the factor of 62.5 (minAP2). The NP determined with the standard $\mathrm{ABA}$ test was $71.5 \mathrm{~kg} \mathrm{CaCO}_{3} / \mathrm{t}$, the carbNP calculated from the carbonate $\mathrm{C}$ concentration was $68.3 \mathrm{~kg} \mathrm{CaCO}_{3} / \mathrm{t}$ and the minNP calculated by mineralogy was $59.4 \mathrm{~kg} \mathrm{CaCO}_{3} / \mathrm{t}$. Based on the calculations, the main three minNP-contributing minerals were dolomite $\left(48.4 \mathrm{~kg} \mathrm{CaCO}_{3} / \mathrm{t}\right)$, biotite $\left(8.75 \mathrm{~kg} \mathrm{CaCO}_{3} / \mathrm{t}\right)$ and talc $(2.33 \mathrm{~kg} \mathrm{CaCO} / \mathrm{t})$. The Pampalo waste rock material was classified as non-acid-generating by all the applied ARD prediction methods.

\section{Siilinjärvi}

Drainage water from the Siilinjärvi new waste rock pile had a neutral $\mathrm{pH}(7.0)$ and high alkalinity $(134 \mathrm{mg} / \mathrm{L}$ $\mathrm{CaCO}_{3}$ ). The main minerals were albite $(47.1 \mathrm{wt} \%)$, $\mathrm{k}$ feldspar (15.6 wt \%), biotite (14.6 wt $\%$ ) and aegerineaugite $\left((\mathrm{Ca}, \mathrm{Na})(\mathrm{Mg}, \mathrm{Fe})\left(\mathrm{Si}_{2} \mathrm{O}_{6}\right), 14.4 \mathrm{wt} \%\right)$. The carbonate mineral content was relatively high $(4.41 \mathrm{wt} \%)$, the sample containing $4.41 \mathrm{wt} \%$ of calcite. The total $\mathrm{C}$ concentration was $0.80 \%$ and the carbonate $\mathrm{C}$ concentration $0.76 \%$. The sulphidic mineral content was low (0.34 wt $\%)$, the sample containing $0.22 \mathrm{wt} \%$ of pyrite and $0.12 \mathrm{wt} \%$ of pyrrhotite. The sulphides appeared as single grains, the maximum diameters being a few hundred micrometres but mainly some tens of micrometres. The appearance of the sulphide grains varied, some being unweathered and some oxidised. The amount of detected oxidised Fe sulphides was $0.01 \mathrm{wt} \%$. The total $\mathrm{S}$ concentration was $0.17 \%$ according to the Leco method and $0.17 \%$ according to calculation based on the sulphide mineral amounts. Based on the $\mathrm{S}$ and $\mathrm{C}$ concentrations, the Siilinjärvi new sample was classified into group I.

The NAG $\mathrm{pH}$ of the Siilinjärvi new sample was 10.5, both NAG values ( $\mathrm{pH} 4.5$ and $\mathrm{pH} 7.0$ ) being $0 \mathrm{~kg} \mathrm{H}_{2} \mathrm{SO}_{4} / \mathrm{t}$. The AP calculated based on the total $\mathrm{S}$ and applying the factor of 31.25 was $5.31 \mathrm{~kg}$ $\mathrm{CaCO}_{3} / \mathrm{t}$ (AP1) and $10.6 \mathrm{~kg} \mathrm{CaCO} / \mathrm{t}$ with the factor of 62.5 (AP2). The minAP calculated based on minS 
Table 3 Formulas and molecular weights of selected minerals used in this study (Mineralogy Database 2017)

\begin{tabular}{|c|c|c|}
\hline Mineral & Formula & $\begin{array}{l}\text { Mol. wt. } \\
\text { (g/mol) }\end{array}$ \\
\hline \multicolumn{3}{|l|}{ Carbonates } \\
\hline Calcite & $\mathrm{CaCO}_{3}$ & 100.09 \\
\hline Dolomite & $\mathrm{CaMg}\left(\mathrm{CO}_{3}\right)_{2}$ & 184.4 \\
\hline Magnesite & $\mathrm{MgCO}_{3}$ & 84.31 \\
\hline \multicolumn{3}{|l|}{ Silicates } \\
\hline Actinolite & $\mathrm{Ca}_{2}(\mathrm{Mg}, \mathrm{Fe})_{5} \mathrm{Si}_{8} \mathrm{O}_{22}(\mathrm{OH})_{2}$ & 853.16 \\
\hline Aegerine-augite & $(\mathrm{Ca}, \mathrm{Na})(\mathrm{Mg}, \mathrm{Fe})\left[\mathrm{Si}_{2} \mathrm{O}_{6}\right]$ & 228.05 \\
\hline Almandine & $\mathrm{Fe}_{3} \mathrm{Al}_{2}\left(\mathrm{SiO}_{4}\right)_{3}$ & 497.75 \\
\hline Anorthite & $\mathrm{CaAl}_{2} \mathrm{Si}_{2} \mathrm{O}_{8}$ & 277.41 \\
\hline Anthophyllite & $\mathrm{Mg}_{7}\left(\mathrm{Si}_{8} \mathrm{O}_{22}\right)(\mathrm{OH})_{2}$ & 780.82 \\
\hline Augite & $(\mathrm{Ca}, \mathrm{Na})(\mathrm{Mg}, \mathrm{Fe}, \mathrm{Al}, \mathrm{Ti})(\mathrm{Si}, \mathrm{Al})_{2} \mathrm{O}_{6}$ & 236.35 \\
\hline Biotite & $\mathrm{K}(\mathrm{Mg}, \mathrm{Fe})_{3}\left(\mathrm{AlSi}_{3} \mathrm{O}_{10}\right)(\mathrm{OH}, \mathrm{F})_{2}$ & 433.53 \\
\hline Chlorite $^{\mathrm{a}}$ & $(\mathrm{Mg}, \mathrm{Fe})_{5} \mathrm{Al}\left(\mathrm{Si}_{3} \mathrm{Al}\right) \mathrm{O}_{10}(\mathrm{OH})_{8}$ & 595.22 \\
\hline Chrysotile & $\mathrm{Mg}_{3} \mathrm{Si}_{2} \mathrm{O}_{5}(\mathrm{OH})_{4}$ & 277.11 \\
\hline Diopside & $\mathrm{CaMgSi}_{2} \mathrm{O}_{6}$ & 216.55 \\
\hline Epidote & $\mathrm{Ca}_{2} \mathrm{Al}_{2}\left(\mathrm{Fe}^{3+} ; \mathrm{Al}\right)\left(\mathrm{SiO}_{4}\right)\left(\mathrm{Si}_{2} \mathrm{O}_{7}\right) \mathrm{O}(\mathrm{OH})$ & 519.3 \\
\hline Fe-hornblende & $\mathrm{Ca}_{2}\left[\mathrm{Fe}_{4}(\mathrm{Al}, \mathrm{Fe})\right] \mathrm{Si}_{7} \mathrm{AlO}_{22}(\mathrm{OH})_{2}$ & 947.32 \\
\hline Mg-hornblende & $\mathrm{Ca}_{2}\left[\mathrm{Mg}_{4}(\mathrm{Al}, \mathrm{Fe})\right] \mathrm{Si}_{7} \mathrm{AlO}_{22}(\mathrm{OH})_{2}$ & 821.16 \\
\hline Olivine & $(\mathrm{Mg}, \mathrm{Fe})_{2} \mathrm{SiO}_{4}$ & 153.31 \\
\hline Phlogopite & $\mathrm{KMg}_{3} \mathrm{AlSi}_{3} \mathrm{O}_{10} \mathrm{~F}(\mathrm{OH})$ & 419.25 \\
\hline Serpentine & $(\mathrm{Mg}, \mathrm{Fe})_{3} \mathrm{Si}_{2} \mathrm{O}_{5}(\mathrm{OH})_{4}$ & 300.77 \\
\hline Talc & $\mathrm{Mg}_{3} \mathrm{Si}_{4} \mathrm{O}_{10}(\mathrm{OH})_{2}$ & 379.27 \\
\hline Tremolite & $\mathrm{Ca}_{2} \mathrm{Mg}_{5} \mathrm{Si}_{8} \mathrm{O}_{22}(\mathrm{OH})_{2}$ & 812.37 \\
\hline \multicolumn{3}{|l|}{ Sulphides } \\
\hline Chalcopyrite & $\mathrm{CuFeS}_{2}$ & 183.53 \\
\hline Pentlandite & $(\mathrm{Fe}, \mathrm{Ni})_{9} \mathrm{~S}_{8}$ & 771.94 \\
\hline Pyrite & $\mathrm{FeS}_{2}$ & 119.98 \\
\hline Pyrrhotite & $\mathrm{Fe}_{1-\mathrm{x}} \mathrm{S}$ & 85.12 \\
\hline Sphalerite & $(\mathrm{Zn}, \mathrm{Fe}) \mathrm{S}$ & 96.98 \\
\hline
\end{tabular}

${ }^{a}$ Here, it is assumed that all chlorite is clinochlore

was $4.37 \mathrm{~kg} \mathrm{CaCO}_{3} / \mathrm{t}$ applying the factor of 31.25 (minAP1) and $8.74 \mathrm{~kg} \mathrm{CaCO} / \mathrm{t}$ with the factor of 62.5 (minAP2). The NP according to the standard ABA test was $113 \mathrm{~kg} \mathrm{CaCO}_{3} / \mathrm{t}$, the carbNP calculated from the carbonate $\mathrm{C}$ concentration was $63.3 \mathrm{~kg}$ $\mathrm{CaCO}_{3} / \mathrm{t}$ and the minNP calculated by mineralogy was $63.5 \mathrm{~kg} \mathrm{CaCO}_{3} / \mathrm{t}$. Based on the calculations, the main three minNP-contributing minerals were calcite $\left(46.7 \mathrm{~kg} \mathrm{CaCO}_{3} / \mathrm{t}\right)$, aegerine-augite $(12.66 \mathrm{~kg}$ $\left.\mathrm{CaCO}_{3} / \mathrm{t}\right)$ and biotite $\left(6.74 \mathrm{~kg} \mathrm{CaCO}_{3} / \mathrm{t}\right)$. The Pampalo waste rock material was classified as nonacid-generating by all the applied ARD prediction methods.
Drainage water from the Siilinjärvi old waste rock pile had a lower $\mathrm{pH}$ (6.5) than that from the Siilinjärvi new pile but higher alkalinity $\left(526 \mathrm{mg} / \mathrm{L} \mathrm{CaCO}_{3}\right)$. The main minerals were biotite $(32.8 \mathrm{wt} \%)$, calcite (18.9 wt\%), Fe-hornblende $\left(\mathrm{Ca}_{2}\left[\mathrm{Fe}_{4}(\mathrm{Al}, \mathrm{Fe})\right] \mathrm{Si}_{7} \mathrm{AlO}_{22}(\mathrm{OH})_{2}, 13.5 \mathrm{wt} \%\right)$, phlogopite $\left(\mathrm{KMg}_{3} \mathrm{AlSi}_{3} \mathrm{O}_{10} \mathrm{~F}(\mathrm{OH}), 8.0 \mathrm{wt} \%\right)$ and dolomite $(7.3 \mathrm{wt} \%)$. The carbonate mineral content was high $(26.17 \mathrm{wt} \%)$. The total $\mathrm{C}$ concentration was $2.82 \%$ and the carbonate $\mathrm{C}$ concentration $2.77 \%$. The sulphidic mineral content was low (0.01 wt $\%$ ), the sample containing $0.01 \mathrm{wt} \%$ of pyrrhotite. The sulphides appeared as single grains 
Table 4 Example calculation for the mineralogical NNP and NPR

\begin{tabular}{|c|c|c|c|c|c|c|c|}
\hline Mineral & $\begin{array}{l}\% \text { total } \\
\text { mass }\end{array}$ & Mineral & $\begin{array}{l}\mathrm{wt} \% \mathrm{~S} \text { in } \\
\text { mineral }\end{array}$ & wt $\%$ of mineral in sample & $\begin{array}{l}\text { wt\% } \mathrm{S} \text { in } \\
\text { sample }\end{array}$ & $\begin{array}{l}\min A P 1 \\
(31.25)\end{array}$ & $\begin{array}{l}\min A P 2 \\
(62.5)\end{array}$ \\
\hline Plagioclase & 35.0 & Pyrrhotite & $37.67^{\mathrm{a}}$ & 0.50 & 0.19 & $2.94^{\mathrm{b}}$ & 5.89 \\
\hline Quartz & 25.0 & Pyrite & 53.30 & 0.30 & 0.16 & $5.00^{\mathrm{c}}$ & 9.99 \\
\hline Biotite & 20.0 & Total minAP & & & & 7.94 & 15.88 \\
\hline Mg-Hornblende & 6.0 & & $\begin{array}{l}\mathrm{wt} \% \mathrm{C} \text { in } \\
\text { mineral }\end{array}$ & wt $\%$ of mineral in sample & $\begin{array}{r}\text { wt } \% \mathrm{C} \text { in } \\
\text { sample }\end{array}$ & $\operatorname{minNP}$ & \\
\hline K-feldspar & 6.0 & Calcite & 12.00 & 0.20 & 0.02 & $1.67^{\mathrm{d}}$ & \\
\hline Fe-Hornblende & 4.0 & Biotite & & 20.00 & & $61.94^{\mathrm{e}}$ & \\
\hline Serpentine & 3.0 & Mg-Hornblende & & 6.00 & & $4.90^{\mathrm{f}}$ & \\
\hline Pyrrhotite & 0.5 & Fe-Hornblende & & 4.00 & & $2.83^{\mathrm{g}}$ & \\
\hline Pyrite & 0.3 & Serpentine & & 3.00 & & $6.69^{\mathrm{h}}$ & \\
\hline Calcite & 0.2 & Total minNP & & & & 78.03 & \\
\hline \multirow[t]{3}{*}{ Total } & 100.0 & & & & & & \\
\hline & & & & & NNP & 70.09 & 62.15 \\
\hline & & & & & NPR & 9.83 & 4.91 \\
\hline
\end{tabular}

${ }^{\mathrm{a}}$ wt.\% $\mathrm{S}$ in mineral based on the formula $\mathrm{Fe}_{0.95} \mathrm{~S}$ for pyrrhotite

${ }^{\mathrm{b}} 0.19(\mathrm{wt} \% \mathrm{~S}) \times 31.25 / 2$, as the oxidation of pyrrhotite via oxygen produces only half the amount of $\mathrm{H}^{+}$compared to pyrite

${ }^{\mathrm{c}} 0.16(\mathrm{wt} \% \mathrm{~S}) \times 31.25$

d $0.02(\mathrm{wt} \% \mathrm{C}) \times 83.3$

e $20(\mathrm{wt} \%) / 100 \times 1000 \mathrm{~kg} / \mathrm{t} \times 100.09(\mathrm{~g} / \mathrm{mol}) / 216.55(\mathrm{~g} / \mathrm{mol}) \times 0.67$

${ }^{\mathrm{f}} 6(\mathrm{wt} \%) / 100 \times 1000 \mathrm{~kg} / \mathrm{t} \times 100.09(\mathrm{~g} / \mathrm{mol}) / 821.16(\mathrm{~g} / \mathrm{mol}) \times 0.67$

$\mathrm{g}_{4}(\mathrm{wt} \%) / 100 \times 1000 \mathrm{~kg} / \mathrm{t} \times 100.09(\mathrm{~g} / \mathrm{mol}) / 947.32(\mathrm{~g} / \mathrm{mol}) \times 0.67$

${ }^{\mathrm{h}} 3(\mathrm{wt} \%) / 100 \times 1000 \mathrm{~kg} / \mathrm{t} \times 100.09(\mathrm{~g} / \mathrm{mol}) / 300.77(\mathrm{~g} / \mathrm{mol}) \times 0.67$

Table 5 Seepage water $\mathrm{pH}$ and alkalinity measured at mine waste sites

\begin{tabular}{lcc}
\hline Mine site/sample & $\mathrm{pH}$ & $\begin{array}{c}\text { Alkalinity } \\
\mathrm{mg} / \mathrm{L} \mathrm{CaCO}_{3}\end{array}$ \\
\hline Pampalo & 6.7 & 239 \\
Siilinjärvi old & 6.5 & 526 \\
Siilinjärvi new & 7.0 & 134 \\
Horsmanaho old & 7.3 & 87 \\
Horsmanaho new & 7.7 & 610 \\
Kylylahti 2014 & 7.1 & 188 \\
Kylylahti 2017 & 2.9 & - \\
Hitura 2014 & 3.5 & - \\
Hitura 2016 & 4.0 & - \\
Hammaslahti & 3.9 & - \\
Särkiniemi & 3.3 & - \\
Hällinmäki & 6.7 & 8 \\
Laiva & 7.0 & 21 \\
Kevitsa & 7.4 & 100 \\
\hline
\end{tabular}

and as inclusions inside silicates. The sulphide grains were unweathered and small, 20-30 $\mu \mathrm{m}$ at maximum. No oxidised Fe sulphides were detected. The total S concentration was $0.07 \%$ according to the Leco method and $0.004 \%$ according to calculation based on the sulphide mineral amounts. Based on the $\mathrm{S}$ and $\mathrm{C}$ concentrations, the Siilinjärvi old sample was classified into group I.

The NAG pH of the Siilinjärvi old sample was 10.8, both NAG values $(\mathrm{pH} 4.5$ and $\mathrm{pH} 7.0)$ being $0 \mathrm{~kg}$ $\mathrm{H}_{2} \mathrm{SO}_{4} / \mathrm{t}$. The AP calculated based on the total $\mathrm{S}$ and applying the factor of 31.25 was $2.19 \mathrm{~kg} \mathrm{CaCO}_{3} / \mathrm{t}(\mathrm{AP} 1)$ and $4.38 \mathrm{~kg} \mathrm{CaCO}_{3} / \mathrm{t}$ with the factor of 62.5 (AP2). The minAP calculated based on minS was $0.06 \mathrm{~kg} \mathrm{CaCO}_{3} / \mathrm{t}$ applying the factor of 31.25 (minAP1) and $0.12 \mathrm{~kg}$ $\mathrm{CaCO}_{3} / \mathrm{t}$ with the factor of 62.5 (minAP2). The NP according to the standard ABA test delivered was $259 \mathrm{~kg} \mathrm{CaCO} / \mathrm{t}$, the carbNP calculated from the carbonate $\mathrm{C}$ concentration was $231 \mathrm{~kg} \mathrm{CaCO}_{3} / \mathrm{t}$ and the minNP calculated by mineralogy was $290 \mathrm{~kg} \mathrm{CaCO}_{3} / \mathrm{t}$. Based on the calculations, the main three minNP- 


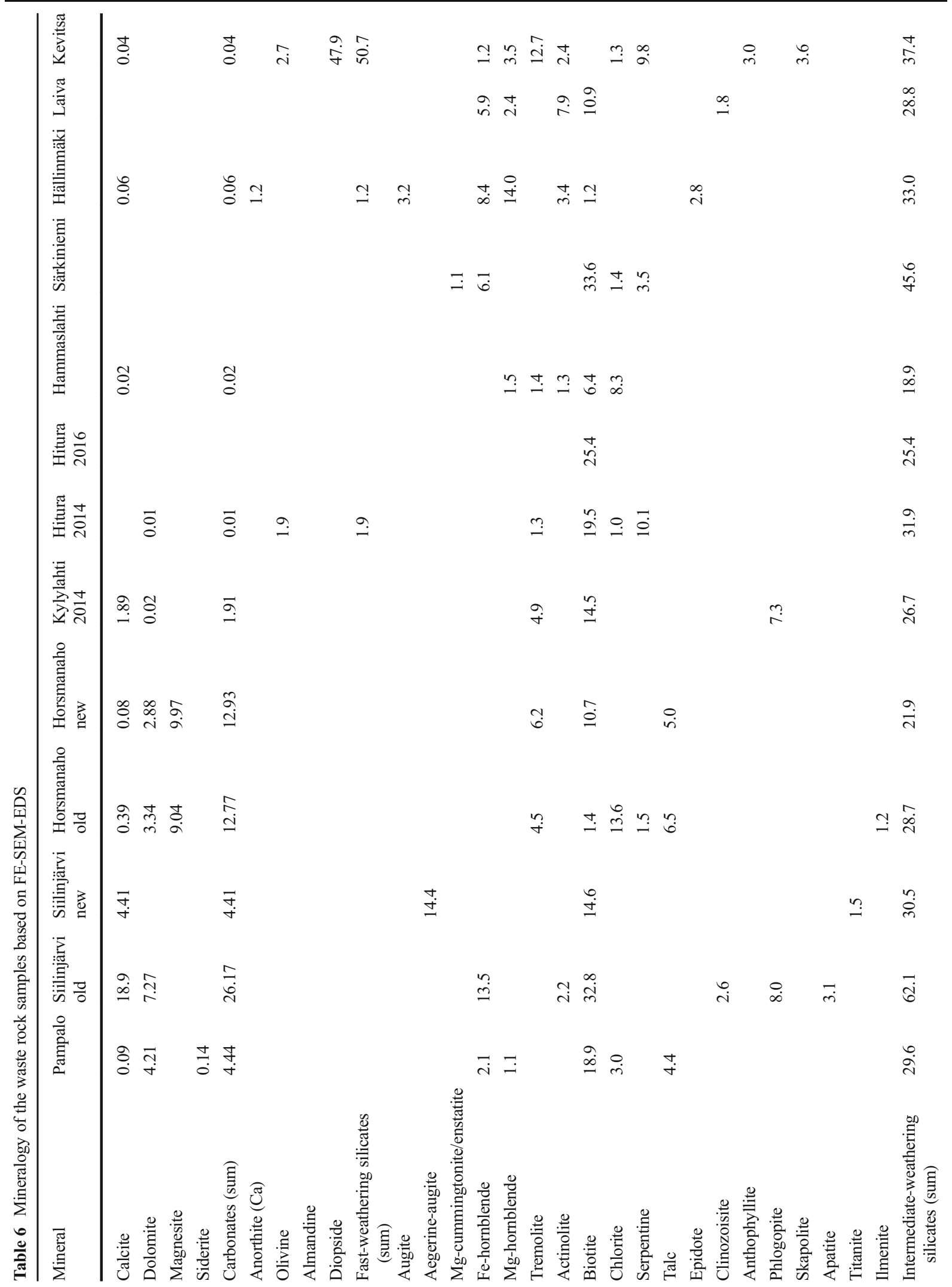




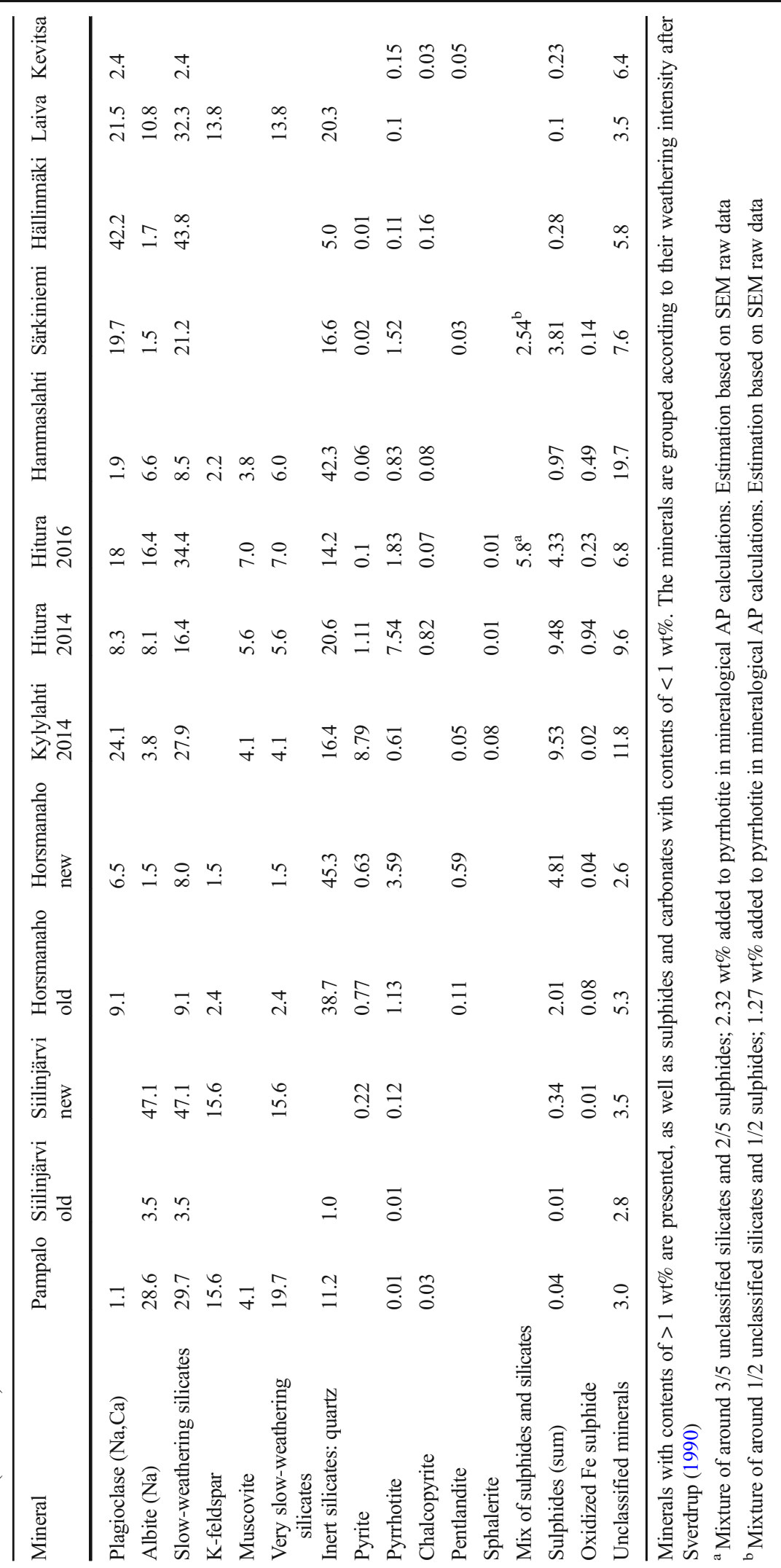




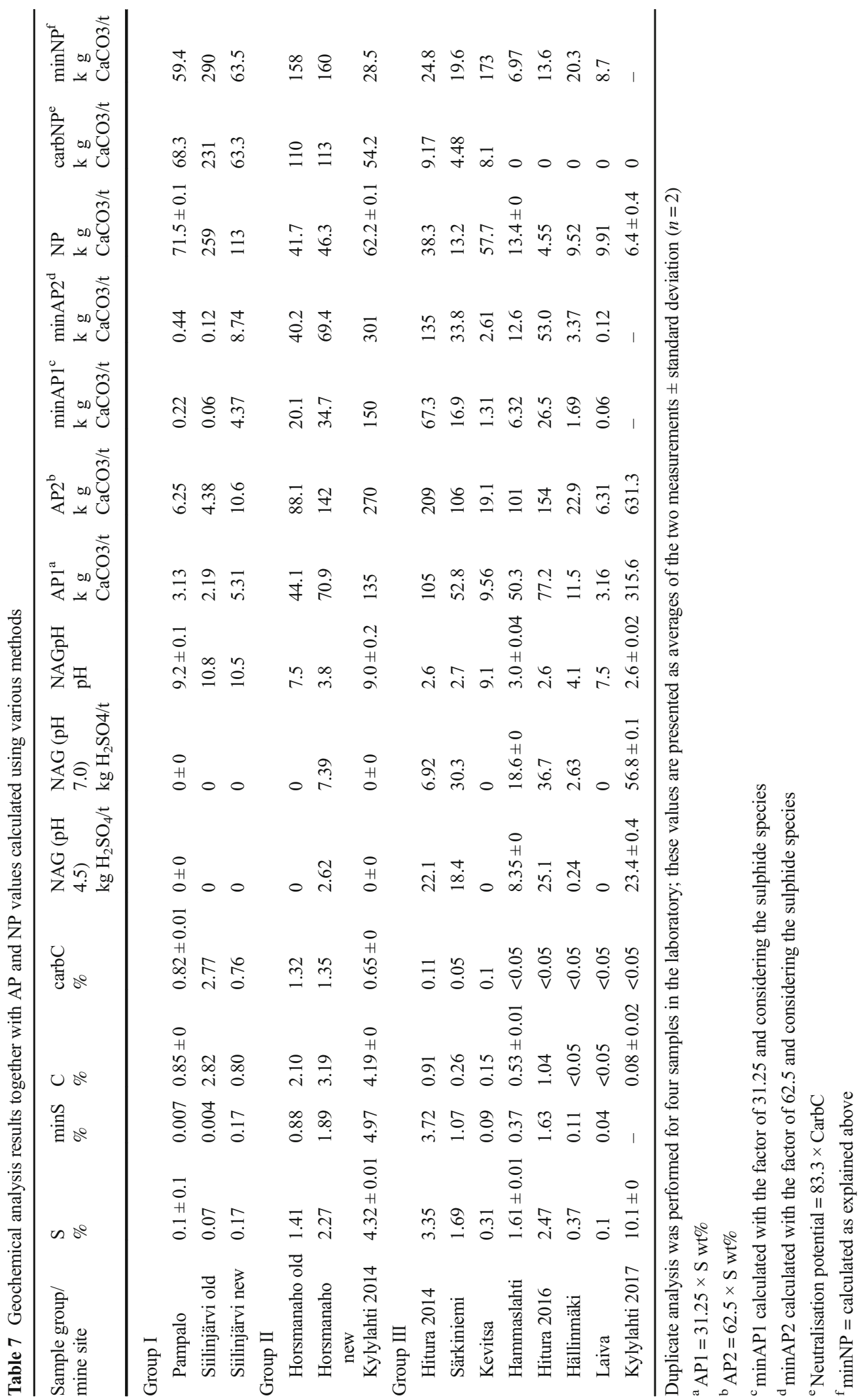


Table 8 Contributions of different minerals to the total minNP of the samples

\begin{tabular}{|c|c|c|c|c|c|c|c|c|c|c|c|c|c|c|}
\hline & Mineral & $\begin{array}{l}\frac{0}{\pi} \\
\frac{0}{0} \\
\frac{1}{\pi} \\
0\end{array}$ & 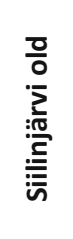 & 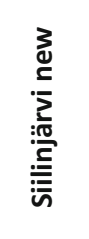 & 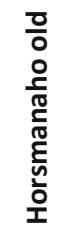 & 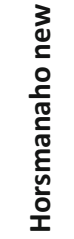 & 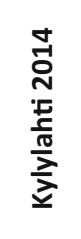 & 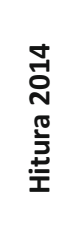 & 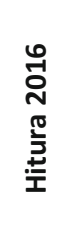 & 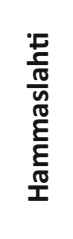 & 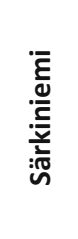 & 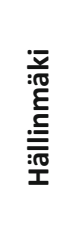 & : & 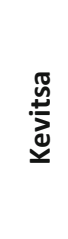 \\
\hline \multirow{4}{*}{ 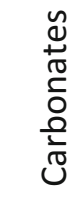 } & Calcite & 0.95 & 20 & 46.7 & 4.13 & 0.85 & 20 & & & 0.21 & & 0.64 & & 0.42 \\
\hline & Dolomite & 48.4 & 83.6 & & 38.4 & 33.1 & 0.23 & 0.12 & & & & & & \\
\hline & Magnesite & & & & 114 & 126 & & & & & & & & \\
\hline & Siderite & 0 & & & & & & & & & & & & \\
\hline \multirow{4}{*}{ 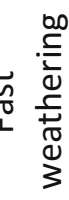 } & Diopside & & & & & & & & & & & & & 148 \\
\hline & Olivine & & & & & & & 8.31 & & & & & & 12 \\
\hline & Anorthite & & & & & & & & & & & 2.95 & & \\
\hline & Almandine & & & & & & & & 1.9 & & & & & \\
\hline \multirow{13}{*}{ 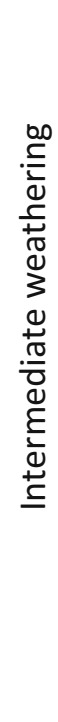 } & Biotite & 8.75 & 15.2 & 6.74 & 0.64 & 4.95 & 5.02 & 8.99 & 11.7 & 2.95 & 15.5 & 0.54 & 5.01 & \\
\hline & Fe-Hornblende & 0.45 & 2.84 & & & & & & & & 1.28 & 1.77 & 1.24 & 0.26 \\
\hline & Mg-Hornblende & 0.27 & & & & & & & & 0.37 & & 3.42 & 0.59 & 0.84 \\
\hline & Tremolite & & & & 1.11 & 1.52 & 0.91 & 0.33 & & 0.35 & & & & 3.12 \\
\hline & Phlogopite & & 3.82 & & & & 3.46 & & & & & & & \\
\hline & Chlorite & 1.04 & & & 4.58 & & & 0.34 & & 2.79 & 0.45 & & & 0.44 \\
\hline & Serpentine & & & & 0.99 & & & 6.7 & & & 2.34 & & & 6.49 \\
\hline & Actinolite & & 0.52 & & & & & & & 0.31 & & 0.8 & 1.85 & \\
\hline & Talc & 2.33 & & & 3.43 & 2.66 & & & & & & & & \\
\hline & $\begin{array}{l}\text { Aegerine- } \\
\text { Augite }\end{array}$ & & & 12.66 & & & & & & & & 9.14 & & \\
\hline & Epidote & & & & & & & & & & & 1.07 & & \\
\hline & Anthophyllite & & & & & & & & & & & & & 0.77 \\
\hline & Total minNP & 62.2 & 306 & 66.1 & 168 & 169 & 29.7 & 24.8 & 13.6 & 6.77 & 19.6 & 20.3 & 8.7 & 173 \\
\hline
\end{tabular}

Values as $\mathrm{kg} \mathrm{CaCO}_{3} /$ t. Highest contribution to NP is presented in dark grey, second highest contribution to NP in medium grey and third highest contribution to NP in light grey

contributing minerals were dolomite $\left(83.6 \mathrm{~kg} \mathrm{CaCO}_{3} / \mathrm{t}\right)$, calcite $\left(20.0 \mathrm{~kg} \mathrm{CaCO}_{3} / \mathrm{t}\right)$ and biotite $\left(15.2 \mathrm{~kg} \mathrm{CaCO}_{3} / \mathrm{t}\right)$. The Pampalo waste rock material was classified as nonacid-generating by all the applied ARD prediction methods.

\section{Horsmanaho}

Drainage water from the Horsmanaho new waste rock pile had a neutral $\mathrm{pH}(7.7)$ and high alkalinity $(610 \mathrm{mg} / \mathrm{L}$ $\left.\mathrm{CaCO}_{3}\right)$. The main minerals were quartz $(45.3 \mathrm{wt} \%)$, biotite (10.7 wt $\%)$, magnesite (9.97 wt\%), plagioclase (other than albite, $6.5 \mathrm{wt} \%$ ), tremolite $(6.2 \mathrm{wt} \%$ ) and talc $\left(\mathrm{Mg}_{3} \mathrm{Si}_{4} \mathrm{O}_{10}(\mathrm{OH})_{2}, 5.0 \mathrm{wt} \%\right)$. The carbonate mineral content was relatively high (12.93 wt.\%), the sample containing $9.97 \mathrm{wt} \%$ of magnesite, $3.34 \mathrm{wt} \%$ of dolomite and $0.39 \mathrm{wt} \%$ of calcite. The total $\mathrm{C}$ concentration was $3.19 \%$ and the carbonate $\mathrm{C}$ concentration $1.35 \%$. The sulphidic mineral content was $4.81 \mathrm{wt} \%$, the sample containing $3.59 \mathrm{wt} \%$ of pyrrhotite, $0.63 \mathrm{wt} \%$ of pyrite and $0.59 \mathrm{wt} \%$ of pentlandite $\left((\mathrm{Fe}, \mathrm{Ni})_{9} \mathrm{~S}_{8}\right)$. The sulphides commonly appeared as inclusions inside 
Table 9 NNP and NPR values calculated with different APs and NPs and compared with the drainage $\mathrm{pH}$

\begin{tabular}{|c|c|c|c|c|c|c|c|c|c|c|c|}
\hline Mine site & $\begin{array}{c}\text { NNP } \\
\text { NP-AP1 } \\
\end{array}$ & $\begin{array}{c}\text { NPR } \\
\text { NP/AP1 }\end{array}$ & $\begin{array}{c}\text { NNP } \\
\text { minNP- } \\
\text { minAP1 }\end{array}$ & $\begin{array}{c}\text { NPR } \\
\operatorname{minNP/} \\
\min A P 1 \\
\end{array}$ & $\begin{array}{c}\text { NNP } \\
\text { minNP- } \\
\text { AP1 } \\
\end{array}$ & $\begin{array}{c}\text { NPR } \\
\text { minNP/ } \\
\text { AP1 }\end{array}$ & $\begin{array}{c}\text { NNP } \\
\text { carbNP } \\
\text {-AP1 } \\
\end{array}$ & $\begin{array}{c}\text { NPR } \\
\text { carbNP } \\
\text { /AP1 }\end{array}$ & $\begin{array}{c}\text { NNP } \\
\text { carbNP- } \\
\text { minAP1 }\end{array}$ & $\begin{array}{c}\text { NPR } \\
\text { carbNP } \\
/ \operatorname{minAP1} \\
\end{array}$ & Drainage \\
\hline \multicolumn{12}{|l|}{ Group I } \\
\hline Pampalo & 68 & 23 & 59 & 270 & 56 & 19 & 65 & 22 & 68 & 311 & 6.7 \\
\hline Siilinjärvi old & 257 & 118 & 290 & 4838 & 288 & 133 & 229 & 106 & 231 & 3848 & 6.5 \\
\hline Siilinjärvi new & 108 & 21 & 59 & 15 & 58 & 12 & 58 & 12 & 59 & 15 & 7.0 \\
\hline \multicolumn{12}{|l|}{ Group II } \\
\hline Horsmanaho old & -2.4 & 0.9 & 138 & 7.9 & 114 & 3.6 & 66 & 2.5 & 90 & 5.5 & 7.3 \\
\hline Horsmanaho new & -25 & 0.7 & 125 & 4.6 & 89 & 2.2 & 42 & 1.6 & 78 & 3.2 & 7.7 \\
\hline Kylylahti 2014 & -73 & 0.5 & -122 & 0.2 & -107 & 0.2 & -81 & 0.4 & -96 & 0.4 & 7.1 \\
\hline \multicolumn{12}{|l|}{ Group III } \\
\hline Hitura 2014 & -66 & 0.4 & -43 & 0.4 & -80 & 0.2 & -96 & 0.1 & -58 & 0.1 & 3.5 \\
\hline Särkiniemi & -40 & 0.2 & 2.7 & 1.2 & -33 & 0.4 & -48 & 0.1 & -12 & 0.3 & 3.3 \\
\hline Kevitsa & 48 & 6.0 & 171 & 132 & 164 & 18 & -1.5 & 0.8 & 6.8 & 6.2 & 7.4 \\
\hline Hammaslahti & -37 & 0.3 & 0.6 & 1.1 & -43 & 0.1 & -50 & 0.0 & -6.3 & 0.0 & 3.9 \\
\hline Hitura 2016 & -73 & 0.1 & -13 & 0.5 & -64 & 0.2 & -77 & 0.0 & -27 & 0.0 & 4.0 \\
\hline Hällinmäki & -1.9 & 0.8 & 18 & 12 & 8.8 & 1.8 & -12 & 0.0 & -1.7 & 0.0 & 6.7 \\
\hline Laiva & 6.8 & 3.1 & 8.6 & 145 & 5.5 & 2.8 & -3.2 & 0.0 & -0.1 & 0.0 & 7.0 \\
\hline Kylylahti 2017 & -309 & 0.02 & - & - & - & - & -316 & 0.0 & - & - & 2.9 \\
\hline
\end{tabular}

AP1 factor $=31.25$. Non-acid-generating is presented in white cells, potentially acid-generating in dark grey and uncertainty zone in light grey

silicates. The sulphide grains were mainly unweathered and small, primarily in the size fraction of 10-20 $\mu \mathrm{m}$. The amount of detected oxidised Fe sulphides was $0.04 \mathrm{wt} \%$. The total S concentration was $2.27 \%$ according to the Leco method and $1.89 \%$ according to calculation based on the sulphide mineral amounts. Based on the $\mathrm{S}$ and $\mathrm{C}$ concentrations, the Horsmanaho new sample was classified into group II.

The NAG $\mathrm{pH}$ of the Horsmanaho new sample was 3.8 , and the NAG values were $2.62 \mathrm{~kg} \mathrm{H}_{2} \mathrm{SO}_{4} / \mathrm{t}(\mathrm{pH} 4.5)$ and $7.39 \mathrm{~kg} \mathrm{H}_{2} \mathrm{SO}_{4} / \mathrm{t}$ (pH 7.0). The AP calculated based on the total $\mathrm{S}$ and applying the factor of 31.25 was $70.9 \mathrm{~kg} \mathrm{CaCO}_{3} / \mathrm{t}$ (AP1) and $142 \mathrm{~kg} \mathrm{CaCO} / \mathrm{t}$ with the factor of 62.5 (AP2). The minAP calculated based on minS was $34.7 \mathrm{~kg} \mathrm{CaCO}_{3} / \mathrm{t}$ applying the factor of 31.25 (minAP1) and $69.4 \mathrm{~kg} \mathrm{CaCO}_{3} / \mathrm{t}$ with the factor of 62.5 (minAP2). The NP according to the standard ABA test was $46.3 \mathrm{~kg} \mathrm{CaCO}_{3} / \mathrm{t}$, the carbNP calculated from the carbonate $\mathrm{C}$ concentration was $113 \mathrm{~kg} \mathrm{CaCO}_{3} / \mathrm{t}$ and the minNP calculated by mineralogy was $160 \mathrm{~kg} \mathrm{CaCO} / \mathrm{t}$. Based on the calculations, the main three minNPcontributing minerals were magnesite $\left(126 \mathrm{~kg} \mathrm{CaCO}_{3} /\right.$ t), dolomite $\left(33.1 \mathrm{~kg} \mathrm{CaCO}_{3} / \mathrm{t}\right)$ and biotite $(4.95 \mathrm{~kg}$ $\mathrm{CaCO}_{3} / \mathrm{t}$ ). The Horsmanaho new waste rock material was classified as non-acid-generating by most of the applied ARD prediction methods.

Drainage water from the Horsmanaho old waste rock pile had a lower $\mathrm{pH}$ (7.3) than that from the Horsmanaho new pile and lower alkalinity $\left(87 \mathrm{mg} / \mathrm{L} \mathrm{CaCO}_{3}\right)$. The main minerals were quartz $(38.7 \mathrm{wt} \%)$, chlorite $\left((\mathrm{Mg}, \mathrm{Fe})_{5} \mathrm{Al}\left(\mathrm{Si}_{3} \mathrm{Al}\right) \mathrm{O}_{10}(\mathrm{OH})_{8}, 13.6 \mathrm{wt} \%\right)$, plagioclase (other than albite, $9.1 \mathrm{wt} \%$ ), magnesite (9.04 wt \%) and talc $(6.5 \mathrm{wt} \%)$. Some traces of gypsum were detected (silicate-gypsum mixture $0.02 \mathrm{wt} \%$ ). The carbonate mineral content was high $(12.77 \mathrm{wt} \%)$. The total $\mathrm{C}$ concentration was $2.10 \%$ and the carbonate $\mathrm{C}$ concentration $1.32 \%$. The sulphidic mineral content was $2.01 \mathrm{wt} \%$, the sample containing $1.13 \mathrm{wt} \%$ of pyrrhotite, $0.77 \mathrm{wt} \%$ of pyrite and $0.11 \mathrm{wt} \%$ of pentlandite. The sulphides appeared as single grains, primarily in the size fraction of 10-20 $\mu \mathrm{m}$. The appearance of the sulphide grains varied, some being unweathered and some strongly oxidised. The amount of detected oxidised $\mathrm{Fe}$ sulphides was $0.08 \mathrm{wt} \%$. The total $\mathrm{S}$ concentration was $1.41 \%$ according to the Leco method and $0.88 \%$ according to calculation based on the sulphide mineral amounts. Based on the $\mathrm{S}$ and $\mathrm{C}$ concentrations, the Horsmanaho old sample was classified into group II. 
Table 10 NNP and NPR values calculated with different APs and NPs and compared with the drainage $\mathrm{pH}$

Table 10 NNP and NPR values calculated with different APs and NPs and compared with the drainage $\mathrm{pH}$. AP2 factor $=62.5$

\begin{tabular}{|c|c|c|c|c|c|c|c|c|c|c|c|}
\hline Mine site & $\begin{array}{c}\text { NNP } \\
\text { NP-AP2 }\end{array}$ & $\begin{array}{c}\text { NPR } \\
\text { NP/AP2 }\end{array}$ & $\begin{array}{c}\text { NNP } \\
\text { minNP- } \\
\min A P 2\end{array}$ & $\begin{array}{c}\text { NPR } \\
\operatorname{minNP} / \\
\min A P 2\end{array}$ & $\begin{array}{l}\text { NNP } \\
\operatorname{minNP} \\
-\mathrm{AP2} \\
\end{array}$ & $\begin{array}{c}\text { NPR } \\
\text { minNP } \\
\text { /AP2 }\end{array}$ & $\begin{array}{c}\text { NNP } \\
\text { carbNP } \\
-\mathrm{AP} 2 \\
\end{array}$ & $\begin{array}{c}\text { NPR } \\
\text { carbNP } \\
\text { /AP2 }\end{array}$ & $\begin{array}{c}\text { NNP } \\
\text { carbNP } \\
\text {-minAP2 }\end{array}$ & $\begin{array}{c}\text { NPR } \\
\text { carbNP } \\
/ \min A P 2\end{array}$ & Drainage \\
\hline \multicolumn{12}{|l|}{ Group I } \\
\hline Pampalo & 65 & 11 & 59 & 135 & 53 & 9.5 & 62 & 11 & 68 & 155 & 6.7 \\
\hline Siilinjärvi old & 255 & 59 & 290 & 2419 & 286 & 66 & 226 & 53 & 231 & 1924 & 6.5 \\
\hline Siilinjärvi new & 102 & 11 & 55 & 7.3 & 53 & 6.0 & 53 & 6.0 & 55 & 7.2 & 7.0 \\
\hline \multicolumn{12}{|l|}{ Group II } \\
\hline Horsmanaho old & -46 & 0.5 & 118 & 3.9 & 70 & 1.8 & 22 & 1.2 & 70 & 2.7 & 7.3 \\
\hline Horsmanaho new & -96 & 0.3 & 90 & 2.3 & 18 & 1.1 & -29 & 0.8 & 43 & 1.6 & 7.7 \\
\hline Kylylahti 2014 & -208 & 0.2 & -272 & 0.1 & -242 & 0.1 & -216 & 0.2 & -246 & 0.2 & 7.1 \\
\hline \multicolumn{12}{|l|}{ Group III } \\
\hline Hitura 2014 & -171 & 0.2 & -110 & 0.2 & -185 & 0.1 & -200 & 0.0 & -126 & 0.1 & 3.5 \\
\hline Särkiniemi & -92 & 0.1 & -14 & 0.6 & -86 & 0.2 & -101 & 0.0 & -29 & 0.1 & 3.3 \\
\hline Kevitsa & 39 & 3.0 & 171 & 66 & 154 & 9.1 & -11 & 0.4 & 5.5 & 3.1 & 7.4 \\
\hline Hammaslahti & -87 & 0.1 & -5.7 & 0.6 & -94 & 0.1 & -101 & 0.0 & -13 & 0.0 & 3.9 \\
\hline Hitura 2016 & -150 & 0.0 & -39 & 0.3 & -141 & 0.1 & -154 & 0.0 & -53 & 0.0 & 4.0 \\
\hline Hällinmäki & -13 & 0.4 & 17 & 6.0 & -2.7 & 0.9 & -23 & 0.0 & -3.4 & 0.0 & 6.7 \\
\hline Laiva & 3.6 & 1.6 & 8.6 & 73 & 2.4 & 1.4 & -6.3 & 0.0 & -0.1 & 0.0 & 7.0 \\
\hline Kylylahti 2017 & -625 & 0.01 & - & - & - & - & -631 & 0.0 & - & - & 2.9 \\
\hline
\end{tabular}

The NAG pH of the Horsmanaho old sample was 7.5, both $\mathrm{NAG}$ values ( $\mathrm{pH} 4.5$ and $\mathrm{pH} 7.0$ ) being $0 \mathrm{~kg} \mathrm{H}_{2} \mathrm{SO}_{4} /$ t. The AP calculated based on the total $\mathrm{S}$ and applying the factor of 31.25 was $44.1 \mathrm{~kg} \mathrm{CaCO}_{3} / \mathrm{t}(\mathrm{AP} 1)$ and $88.1 \mathrm{~kg}$ $\mathrm{CaCO}_{3} / \mathrm{t}$ with the factor of 62.5 (AP2). The minAP calculated based on minS was $20.1 \mathrm{~kg} \mathrm{CaCO}_{3} / \mathrm{t}$ applying the factor of 31.25 (minAP1) and $40.2 \mathrm{~kg} \mathrm{CaCO}_{3} / \mathrm{t}$ with the factor of 62.5 (minAP2). The NP according to the standard $\mathrm{ABA}$ test was $41.7 \mathrm{~kg} \mathrm{CaCO}_{3} / \mathrm{t}$, the carbNP calculated from the carbonate $\mathrm{C}$ concentration was $110 \mathrm{~kg} \mathrm{CaCO}_{3} / \mathrm{t}$ and the minNP calculated by mineralogy was $158 \mathrm{~kg} \mathrm{CaCO}_{3} / \mathrm{t}$. Based on the calculations, the main three minNP-contributing minerals were magnesite $\left(114 \mathrm{~kg} \mathrm{CaCO}_{3} / \mathrm{t}\right)$, dolomite $\left(38.4 \mathrm{~kg} \mathrm{CaCO}_{3} / \mathrm{t}\right)$ and chlorite $\left(4.58 \mathrm{~kg} \mathrm{CaCO}_{3} / \mathrm{t}\right)$. The Horsmanaho old waste rock material was classified as non-acid-generating by most of the applied ARD prediction methods.

\section{Kylylahti}

Drainage water from the Kylylahti waste rock pile had a neutral $\mathrm{pH}$ (7.1) and high alkalinity $\left(188 \mathrm{mg} / \mathrm{L} \mathrm{CaCO}_{3}\right)$ in 2014. When the drainage was measured again in 2017 , the $\mathrm{pH}$ had dropped to 2.9 and the alkalinity could not be measured. The main minerals of the Kylylahti 2014 sample were plagioclase (other than albite, $24.1 \mathrm{wt} \%)$, quartz (16.4 wt $\%)$, biotite (14.5 wt\%), phlogopite $(7.3 \mathrm{wt} \%)$ and pyrite $(8.79 \mathrm{wt} \%)$. Some traces of gypsum were detected (silicate-gypsum mixture $0.01 \mathrm{wt} \%$ ). The Kylylahti 2014 sample contained a substantial amount of unclassified minerals (11.8 wt $\%$ ). The carbonate mineral content was $1.91 \mathrm{wt} \%$, the sample containing $1.89 \mathrm{wt} \%$ of calcite and $0.02 \mathrm{wt} \%$ of dolomite. The total C concentration was $4.19 \%$ and the carbonate $\mathrm{C}$ concentration $0.65 \%$. The sulphidic mineral content was $9.53 \mathrm{wt} \%$, the sample containing $8.79 \mathrm{wt} \%$ of pyrite, $0.61 \mathrm{wt} \%$ of pyrrhotite, $0.08 \mathrm{wt} \%$ of sphalerite and $0.05 \mathrm{wt} \%$ of pentlandite. The sulphides appeared as single grains, pyrite primarily in the size fraction of $<20 \mu \mathrm{m}$ and pyrrhotite in the size fraction of $10-40 \mu \mathrm{m}$. The sulphide grains were mainly unweathered, but some grains were slightly or some strongly oxidised. The amount of detected oxidised Fe sulphides was $0.02 \mathrm{wt} \%$. The total $\mathrm{S}$ concentration was $4.32 \%$ 
according to the Leco method and $4.97 \%$ according to calculation based on the sulphide mineral amounts. Based on the $\mathrm{S}$ and $\mathrm{C}$ concentrations, the Kylylahti 2014 sample was classified into group II.

The NAG pH of the Kylylahti 2014 sample was 9.0, both NAG values ( $\mathrm{pH} 4.5$ and $\mathrm{pH} 7.0$ ) being $0 \mathrm{~kg}$ $\mathrm{H}_{2} \mathrm{SO}_{4} / \mathrm{t}$. The AP calculated based on the total $\mathrm{S}$ and applying the factor of 31.25 was $135 \mathrm{~kg} \mathrm{CaCO}_{3} / \mathrm{t}$ (AP1) and $270 \mathrm{~kg} \mathrm{CaCO}_{3} / \mathrm{t}$ with the factor of 62.5 (AP2). The minAP calculated based on minS was $150 \mathrm{~kg} \mathrm{CaCO}_{3} / \mathrm{t}$ applying the factor of 31.25 (minAP1) and $301 \mathrm{~kg}$ $\mathrm{CaCO}_{3} / \mathrm{t}$ with the factor of 62.5 (minAP2). The NP according to the standard ABA test was $62.2 \mathrm{~kg}$ $\mathrm{CaCO}_{3} / \mathrm{t}$, the carbNP calculated from the carbonate $\mathrm{C}$ concentration was $54.2 \mathrm{~kg} \mathrm{CaCO}_{3} / \mathrm{t}$ and the minNP calculated by mineralogy was $28.5 \mathrm{~kg} \mathrm{CaCO}_{3} / \mathrm{t}$. Based on the calculations, the main three minNP-contributing minerals were calcite $\left(20 \mathrm{~kg} \mathrm{CaCO}_{3} / \mathrm{t}\right)$, biotite $(5.02 \mathrm{~kg}$ $\left.\mathrm{CaCO}_{3} / \mathrm{t}\right)$ and phlogopite $\left(3.46 \mathrm{~kg} \mathrm{CaCO}_{3} / \mathrm{t}\right)$. The Kylylahti 2014 waste rock material was classified as acid-generating by all the applied ARD prediction methods, except NAG $\mathrm{pH}$, which predicted non-acid generation.

\section{Hitura}

In 2014, drainage water from the Hitura waste rock pile had an acid pH (3.5) and the alkalinity could not be measured. In the Hitura 2014 sample, the main minerals were quartz (20.6 wt\%), biotite (19.5 wt\%), serpentine (10.1 wt $\%$ ), plagioclase (other than albite, $8.3 \mathrm{wt} \%$ ), albite (8.1 wt \%), pyrrhotite (7.54 wt\%) and muscovite $\left(\left(\mathrm{KAl}_{2}\left(\mathrm{Si}_{3} \mathrm{Al}\right) \mathrm{O}_{10}(\mathrm{OH}, \mathrm{F})_{2}\right), 5.6 \mathrm{wt} \%\right)$. The carbonate mineral content was small, and only $0.01 \mathrm{wt} \%$ of dolomite was detected. The total $\mathrm{C}$ concentration was $0.91 \%$ and the carbonate $\mathrm{C}$ concentration $0.11 \%$. The sulphidic mineral content was $9.48 \mathrm{wt} \%$, the sample containing $7.54 \mathrm{wt} \%$ of pyrrhotite, $1.11 \mathrm{wt} \%$ of pyrite, $0.82 \mathrm{wt} \%$ of chalcopyrite and $0.01 \mathrm{wt} \%$ of sphalerite. The sulphides appeared as single grains, primarily in the size fraction of 10-20 $\mu \mathrm{m}$. The appearance of the sulphide grains varied, most being unweathered and some oxidised. The amount of detected oxidised Fe sulphides was $0.94 \mathrm{wt} \%$. The total $\mathrm{S}$ concentration was $3.35 \%$ according to the Leco method and $3.72 \%$ according to calculation based on the sulphide mineral amounts. Based on the $\mathrm{S}$ and $\mathrm{C}$ concentrations, the Hitura 2014 sample was classified into group III.
The NAG pH of the Hitura 2014 sample was 2.6, and the $\mathrm{NAG}$ values were $22.1 \mathrm{~kg} \mathrm{H}_{2} \mathrm{SO}_{4} / \mathrm{t}$ ( $\mathrm{pH} \mathrm{4.5)}$ and $6.92 \mathrm{~kg} \mathrm{H}_{2} \mathrm{SO}_{4} / \mathrm{t}$ ( $\left.\mathrm{pH} 7.0\right)$. The AP calculated based on the total S and applying the factor of 31.25 was $105 \mathrm{~kg}$ $\mathrm{CaCO}_{3} / \mathrm{t}(\mathrm{AP} 1)$ and $109 \mathrm{~kg} \mathrm{CaCO}_{3} / \mathrm{t}$ with the factor of 62.5 (AP2). The minAP calculated based on minS was $67.3 \mathrm{~kg} \mathrm{CaCO}_{3} / \mathrm{t}$ applying the factor of 31.25 (minAP1) and $135 \mathrm{~kg} \mathrm{CaCO}_{3} / \mathrm{t}$ with the factor of 62.5 (minAP2). The NP according to the standard ABA test was $38.3 \mathrm{~kg}$ $\mathrm{CaCO}_{3} / \mathrm{t}$, the carbNP calculated from the carbonate $\mathrm{C}$ concentration was $9.17 \mathrm{~kg} \mathrm{CaCO}_{3} / \mathrm{t}$ and the minNP calculated by mineralogy was $24.8 \mathrm{~kg} \mathrm{CaCO} / \mathrm{t}$. Based on the calculations, the main three minNP-contributing minerals were biotite $\left(8.99 \mathrm{~kg} \mathrm{CaCO}_{3} / \mathrm{t}\right)$, olivine $\left(8.31 \mathrm{~kg} \mathrm{CaCO}_{3} / \mathrm{t}\right)$ and serpentine $\left(6.70 \mathrm{~kg} \mathrm{CaCO}_{3} / \mathrm{t}\right)$. The Hitura 2014 waste rock material was classified as acid-generating by all the applied ARD prediction methods.

In 2016, drainage water from the Hitura waste rock pile had a $\mathrm{pH}$ of 4.0 , which was slightly less acid than in 2014. The alkalinity could not be measured. In the Hitura 2016 sample, the main minerals were biotite (25.4 wt\%), plagioclase (other than albite, $18.0 \mathrm{wt} \%$ ), albite (16.4 wt\%), quartz (14.2 wt\%) and muscovite (7.0 wt \%). No carbonates were detected. The total C concentration was $1.04 \%$ and the carbonate $\mathrm{C}$ concentration $<0.05 \%$. The sulphidic mineral content was $4.33 \mathrm{wt} \%$, the sample containing $1.83 \mathrm{wt} \%$ of pyrrhotite, $0.10 \mathrm{wt} \%$ of pyrite, $0.07 \mathrm{wt} \%$ of chalcopyrite, $0.01 \mathrm{wt} \%$ of sphalerite and a mixture of sulphides and silicates ( $5.8 \mathrm{wt} \%$ ), which was calculated as $2.32 \mathrm{wt} \%$ of pyrrhotite based on the SEM data. The sulphides appeared as single grains, pyrrhotite primarily in the size fraction of $<30 \mu \mathrm{m}$ and pyrite in the size fraction of $<$ $20 \mu \mathrm{m}$. The appearance of the sulphide grains varied, some being unweathered and some oxidised. The amount of detected oxidised Fe sulphides was $0.23 \mathrm{wt} \%$. The total S concentration was $2.47 \%$ according to the Leco method and $1.63 \%$ according to calculation based on the sulphide mineral amounts. Based on the $\mathrm{S}$ and $\mathrm{C}$ concentrations, the Hitura 2016 sample was classified into group III.

The NAG pH of the Hitura 2016 sample was 2.6, and the NAG values were $25.1 \mathrm{~kg} \mathrm{H}_{2} \mathrm{SO}_{4} / \mathrm{t}(\mathrm{pH} 4.5)$ and $36.7 \mathrm{~kg} \mathrm{H}_{2} \mathrm{SO}_{4} / \mathrm{t}$ ( $\mathrm{pH} 7.0$ ). The AP calculated based on the total S and applying the factor of 31.25 was $77.2 \mathrm{~kg}$ $\mathrm{CaCO}_{3} / \mathrm{t}(\mathrm{AP} 1)$ and $154 \mathrm{~kg} \mathrm{CaCO}_{3} / \mathrm{t}$ with the factor of 62.5 (AP2). The minAP calculated based on minS was $26.5 \mathrm{~kg} \mathrm{CaCO}_{3} / \mathrm{t}$ applying the factor of 31.25 (minAP1) 
and $53 \mathrm{~kg} \mathrm{CaCO}_{3} / \mathrm{t}$ with the factor of 62.5 (minAP2). The NP according to the standard ABA test was $4.55 \mathrm{~kg}$ $\mathrm{CaCO}_{3} / \mathrm{t}$, the carbNP calculated from the carbonate $\mathrm{C}$ concentration was $0 \mathrm{~kg} \mathrm{CaCO} / \mathrm{t}$ and the minNP calculated by mineralogy was $13.6 \mathrm{~kg} \mathrm{CaCO}_{3} / \mathrm{t}$. Based on the calculations, the main minNP-contributing minerals were biotite $\left(11.7 \mathrm{~kg} \mathrm{CaCO}_{3} / \mathrm{t}\right)$ and almandine $(1.9 \mathrm{~kg}$ $\mathrm{CaCO}_{3} / \mathrm{t}$ ). The Hitura 2016 waste rock material was classified as acid-generating by most of the applied ARD prediction methods.

\section{Hammaslahti}

The water pond below the Hammaslahti waste rock pile had an acid pH (3.9) and the alkalinity could not be measured. The main minerals were quartz (42.3 wt $\%$ ), chlorite $(8.3 \mathrm{wt} \%)$ and biotite $(6.4 \mathrm{wt} \%)$. Some traces of gypsum were detected (silicate-gypsum mixture $0.02 \mathrm{wt} \%$ ). The Hammaslahti sample contained a substantial amount of unclassified minerals (19.7 wt $\%$ ). The carbonate mineral content was low, the sample containing $0.02 \mathrm{wt} \%$ of calcite. The total $\mathrm{C}$ concentration was $0.53 \%$ and the carbonate $\mathrm{C}$ concentration < $0.05 \%$. The sulphidic mineral content was $0.97 \mathrm{wt} \%$, the sample containing $0.83 \mathrm{wt} \%$ of pyrrhotite, $0.08 \mathrm{wt} \%$ of chalcopyrite and $0.06 \mathrm{wt} \%$ of pyrite. The sulphides appeared as single grains, primarily in the size fraction of 10-20 $\mu \mathrm{m}$. The appearance of the sulphide grains varied, most being unweathered and some oxidised. The amount of detected oxidised Fe sulphides was $0.49 \mathrm{wt} \%$. The total $\mathrm{S}$ concentration was $1.61 \%$ according to the Leco method and $0.37 \%$ according to calculation based on the sulphide mineral amounts. Based on the $\mathrm{S}$ and $\mathrm{C}$ concentrations, the Hammaslahti sample was classified into group III.

The NAG pH of the Hammaslahti sample was 3.0, and the NAG values were $8.35 \mathrm{~kg} \mathrm{H}_{2} \mathrm{SO}_{4} / \mathrm{t}(\mathrm{pH} 4.5)$ and $18.6 \mathrm{~kg} \mathrm{H}_{2} \mathrm{SO}_{4} / \mathrm{t}$ ( $\left.\mathrm{pH} 7.0\right)$. The AP calculated based on the total $\mathrm{S}$ and applying the factor of 31.25 was $50.3 \mathrm{~kg}$ $\mathrm{CaCO}_{3} / \mathrm{t}$ (AP1) and $101 \mathrm{~kg} \mathrm{CaCO}_{3} / \mathrm{t}$ with the factor of 62.5 (AP2). The minAP calculated based on $\operatorname{minS}$ was $6.32 \mathrm{~kg} \mathrm{CaCO}_{3} / \mathrm{t}$ applying the factor of 31.25 (minAP1) and $12.6 \mathrm{~kg} \mathrm{CaCO}_{3} / \mathrm{t}$ with the factor of 62.5 (minAP2). The NP according to the standard ABA test was $13.4 \mathrm{~kg}$ $\mathrm{CaCO}_{3} / \mathrm{t}$, the carbNP calculated from the carbonate $\mathrm{C}$ concentration was $0 \mathrm{~kg} \mathrm{CaCO}_{3} / \mathrm{t}$ and the minNP calculated by mineralogy was $6.97 \mathrm{~kg} \mathrm{CaCO} / \mathrm{t}$. Based on the calculations, the main three minNP-contributing minerals were biotite $\left(2.95 \mathrm{~kg} \mathrm{CaCO}_{3} / \mathrm{t}\right)$, chlorite $(2.79 \mathrm{~kg}$
$\left.\mathrm{CaCO}_{3} / \mathrm{t}\right)$ and $\mathrm{Mg}$-hornblende $\left(0.37 \mathrm{~kg} \mathrm{CaCO}_{3} / \mathrm{t}\right)$. The Hammaslahti waste rock material was classified as acidgenerating by most of the applied ARD prediction methods.

\section{Särkiniemi}

Drainage water from the Särkiniemi waste rock pile had an acid $\mathrm{pH}$ (3.3) and the alkalinity could not be measured. The main minerals were biotite $(33.6 \mathrm{wt} \%)$, plagioclase (other than albite, $19.7 \mathrm{wt} \%$ ), quartz (16.6 wt\%) and Fe-hornblende (6.1 wt\%). No carbonates were detected. The total $\mathrm{C}$ concentration was $0.26 \%$ and the carbonate $\mathrm{C}$ concentration $0.05 \%$. The sulphidic mineral content was $3.81 \mathrm{wt} \%$, the sample containing $1.52 \mathrm{wt} \%$ of pyrrhotite, $0.03 \mathrm{wt} \%$ of pentlandite, $0.02 \mathrm{wt} \%$ of pyrite and a mixture of sulphides and silicates (2.54 wt\%), which was calculated as $1.27 \mathrm{wt} \%$ of pyrrhotite based on the SEM data. The sulphides appeared as single grains, primarily in the size fraction of $<30 \mu \mathrm{m}$. The appearance of the sulphide grains varied, some being unweathered and some oxidised. The amount of detected oxidised $\mathrm{Fe}$ sulphides was $0.14 \mathrm{wt} \%$. The total $\mathrm{S}$ concentration was $1.69 \%$ according to the Leco method and $1.07 \%$ according to calculation based on the sulphide mineral amounts. Based on the $\mathrm{S}$ and $\mathrm{C}$ concentrations, the Särkiniemi sample was classified into group III.

The NAG pH of the Särkiniemi sample was 2.7, and the NAG values were $18.4 \mathrm{~kg} \mathrm{H}_{2} \mathrm{SO}_{4} / \mathrm{t}(\mathrm{pH} 4.5)$ and $30.3 \mathrm{~kg} \mathrm{H}_{2} \mathrm{SO}_{4} / \mathrm{t}(\mathrm{pH} \mathrm{7.0)}$. The AP calculated based on the total $\mathrm{S}$ and applying the factor of 31.25 was $52.8 \mathrm{~kg} \mathrm{CaCO}_{3} / \mathrm{t}$ (AP1) and $106 \mathrm{~kg}$ $\mathrm{CaCO}_{3} / \mathrm{t}$ with the factor of 62.5 (AP2). The minAP calculated based on $\operatorname{minS}$ was $16.9 \mathrm{~kg} \mathrm{CaCO}_{3} / \mathrm{t}$ applying the factor of 31.25 (minAP1) and $33.8 \mathrm{~kg} \mathrm{CaCO} / \mathrm{t}$ with the factor of 62.5 (minAP2). The NP according to the standard ABA test was $13.2 \mathrm{~kg} \mathrm{CaCO}_{3} / \mathrm{t}$, the carbNP calculated from the carbonate $\mathrm{C}$ concentration was $4.48 \mathrm{~kg} \mathrm{CaCO}_{3} / \mathrm{t}$ and the minNP calculated by mineralogy was $19.6 \mathrm{~kg}$ $\mathrm{CaCO}_{3} / \mathrm{t}$. Based on the calculations, the main three minNP-contributing minerals were biotite $(15.5 \mathrm{~kg}$ $\left.\mathrm{CaCO}_{3} / \mathrm{t}\right)$, serpentine $(2.34 \mathrm{~kg} \mathrm{CaCO} / \mathrm{t})$ and $\mathrm{Fe}-$ hornblende $(1.28 \mathrm{~kg} \mathrm{CaCO} / \mathrm{t})$. The Särkiniemi waste rock material was classified as acidgenerating by most of the applied ARD prediction methods. 
Hällinmäki

Drainage water from the Hällinmäki waste rock pile had a neutral $\mathrm{pH}$ (6.7) and alkalinity of $8.4 \mathrm{mg} / \mathrm{L} \mathrm{CaCO}_{3}$. The main minerals were plagioclase (other than albite, $42.2 \mathrm{wt} \%$ ), Mg-hornblende $\left(\mathrm{Ca}_{2}\left[\mathrm{Mg}_{4}(\mathrm{Al}, \mathrm{Fe})\right] \mathrm{Si}_{7} \mathrm{AlO}_{22}(\mathrm{OH})_{2}, 14.0 \mathrm{wt} \%\right), \mathrm{Fe}-$ hornblende $(8.4 \mathrm{wt} \%)$ and quartz $(5.0 \mathrm{wt} \%)$. The carbonate mineral content was low, the sample containing $0.06 \mathrm{wt} \%$ of calcite. The total $\mathrm{C}$ concentration was $<0.05 \%$ and the carbonate $\mathrm{C}$ concentration $<0.05 \%$. The sulphidic mineral content was $0.28 \mathrm{wt} \%$, the sample containing $0.16 \mathrm{wt} \%$ of chalcopyrite, $0.11 \mathrm{wt} \%$ of pyrrhotite and $0.01 \mathrm{wt} \%$ of pyrite. The sulphides appeared as single grains and as inclusions inside silicates. The sulphide grains were unweathered and small, mainly just a few micrometres and $40 \mu \mathrm{m}$ at maximum. No oxidized $\mathrm{Fe}$ sulphides were detected. The total $\mathrm{S}$ concentration was $0.37 \%$ according to the Leco method and $0.11 \%$ according to calculation based on the sulphide mineral amounts. Based on the $\mathrm{S}$ and $\mathrm{C}$ concentrations, the Hällinmäki sample was classified into group III.

The NAG pH of the Hällinmäki sample was 4.1, and the NAG values were $0.24 \mathrm{~kg} \mathrm{H}_{2} \mathrm{SO}_{4} / \mathrm{t}(\mathrm{pH} 4.5)$ and $2.63 \mathrm{~kg} \mathrm{H}_{2} \mathrm{SO}_{4} / \mathrm{t}$ ( $\left.\mathrm{pH} 7.0\right)$. The AP calculated based on the total $\mathrm{S}$ and applying the factor of 31.25 was $11.5 \mathrm{~kg}$ $\mathrm{CaCO}_{3} / \mathrm{t}(\mathrm{AP} 1)$ and $22.9 \mathrm{~kg} \mathrm{CaCO}_{3} / \mathrm{t}$ with the factor of 62.5 (AP2). The minAP calculated based on minS was $1.69 \mathrm{~kg} \mathrm{CaCO}_{3} / \mathrm{t}$ applying the factor of 31.25 (minAP1) and $3.37 \mathrm{~kg} \mathrm{CaCO}_{3} / \mathrm{t}$ with the factor of 62.5 (minAP2). The NP according to the standard ABA test was $9.52 \mathrm{~kg}$ $\mathrm{CaCO}_{3} / \mathrm{t}$, the carbNP calculated from the carbonate $\mathrm{C}$ concentration was $0 \mathrm{~kg} \mathrm{CaCO}_{3} / \mathrm{t}$ and the minNP calculated by mineralogy was $20.3 \mathrm{~kg} \mathrm{CaCO}_{3} / \mathrm{t}$. Based on the calculations, the main three minNP-contributing minerals were aegerine-augite $\left(9.14 \mathrm{~kg} \mathrm{CaCO}_{3} / \mathrm{t}\right), \mathrm{Mg}$ hornblende $\left(3.42 \mathrm{~kg} \mathrm{CaCO}_{3} / \mathrm{t}\right)$ and anorthite $(2.95 \mathrm{~kg}$ $\mathrm{CaCO}_{3} / \mathrm{t}$ ). The Hällinmäki waste rock material was classified as acid-generating by most of the applied ARD prediction methods, except when calculating the NPR as $\operatorname{minNP} / \min A P$.

Laiva

Drainage water from the Laiva waste rock pile had a neutral $\mathrm{pH}$ (7.0) and alkalinity of $21.2 \mathrm{mg} / \mathrm{L} \mathrm{CaCO}_{3}$. The main minerals were plagioclase (other than albite,
$21.5 \mathrm{wt} \%)$, quartz (20.3 wt\%), k-feldspar (13.8 wt\%), biotite $(10.9 \mathrm{wt} \%)$, albite $(10.8 \mathrm{wt} \%)$, actinolite $\left(\mathrm{Ca}_{2}(\mathrm{Mg}, \mathrm{Fe})_{5} \mathrm{Si}_{8} \mathrm{O}_{22}(\mathrm{OH})_{2}, 7.9 \mathrm{wt} \%\right)$ and $\mathrm{Fe}-$ hornblende (5.9 wt\%). No carbonates were detected. The total C concentration was $<0.05 \%$ and the carbonate $\mathrm{C}$ concentration $<0.05 \%$. The sulphidic mineral content was low, the sample containing $0.10 \mathrm{wt} \%$ of pyrrhotite. The sulphides appeared as unweathered single grains. The sulphide grains were small, mainly just a few micrometres and $20 \mu \mathrm{m}$ at maximum. No oxidised $\mathrm{Fe}$ sulphides were detected. The total $\mathrm{S}$ concentration was $0.10 \%$ according to the Leco method and $0.04 \%$ according to calculation based on the sulphide mineral amounts. Based on the $\mathrm{S}$ and $\mathrm{C}$ concentrations, the Laiva sample was classified into group III.

The NAG $\mathrm{pH}$ of the Laiva sample was 7.5 , both NAG values (pH 4.5 and $\mathrm{pH} 7.0$ ) being $0 \mathrm{~kg} \mathrm{H}_{2} \mathrm{SO}_{4} / \mathrm{t}$. The AP calculated based on the total $\mathrm{S}$ and applying the factor of 31.25 was $3.16 \mathrm{~kg} \mathrm{CaCO}_{3} / \mathrm{t}$ (AP1) and $6.31 \mathrm{~kg}$ $\mathrm{CaCO}_{3} / \mathrm{t}$ with the factor of 62.5 (AP2). The minAP calculated based on minS was $0.06 \mathrm{~kg} \mathrm{CaCO}_{3} / \mathrm{t}$ applying the factor of 31.25 (minAP1) and $0.12 \mathrm{~kg} \mathrm{CaCO}_{3} / \mathrm{t}$ with the factor of 62.5 (minAP2). The NP according to the standard $\mathrm{ABA}$ test was $9.91 \mathrm{~kg} \mathrm{CaCO}_{3} / \mathrm{t}$, the carbNP calculated from the carbonate $\mathrm{C}$ concentration was $0 \mathrm{~kg}$ $\mathrm{CaCO}_{3} / \mathrm{t}$ and the minNP calculated by mineralogy was $8.70 \mathrm{~kg} \mathrm{CaCO}_{3} / \mathrm{t}$. Based on the calculations, the main three minNP-contributing minerals were biotite $(5.01 \mathrm{~kg}$ $\left.\mathrm{CaCO}_{3} / \mathrm{t}\right)$, actinolite $\left(1.85 \mathrm{~kg} \mathrm{CaCO}_{3} / \mathrm{t}\right)$ and $\mathrm{Fe}$ hornblende $\left(1.24 \mathrm{~kg} \mathrm{CaCO}_{3} / \mathrm{t}\right)$. The applied ARD prediction methods gave conflicting ARD generation predictions for the Laiva waste rock material.

Kevitsa

Drainage water from the Kevitsa waste rock pile had a neutral $\mathrm{pH}$ (7.4) and alkalinity of $100 \mathrm{mg} / \mathrm{L} \mathrm{CaCO}_{3}$. The main minerals were diopside (47.9 wt $\%)$, tremolite (12.7 wt $\%)$ and serpentine $(9.8 \mathrm{wt} \%)$. The carbonate mineral content was low, the sample containing $0.04 \mathrm{wt} \%$ of calcite. The total $\mathrm{C}$ concentration was $0.15 \%$ and the carbonate $\mathrm{C}$ concentration $0.1 \%$. The sulphidic mineral content was $0.23 \mathrm{wt} \%$, the sample containing $0.15 \mathrm{wt} \%$ of pyrrhotite, $0.05 \mathrm{wt} \%$ of pentlandite and $0.03 \mathrm{wt} \%$ of chalcopyrite. The sulphides appeared as unweathered single grains. The sulphide grains were small, mainly just a few micrometres and $40 \mu \mathrm{m}$ at maximum. No oxidised Fe sulphides were detected. The total $\mathrm{S}$ concentration was $0.31 \%$ 
according to the Leco method and $0.09 \%$ according to calculation based on the sulphide mineral amounts. Based on the $\mathrm{S}$ and $\mathrm{C}$ concentrations, the Kevitsa sample was classified into group III.

The NAG pH of the Kevitsa sample was 9.1, both NAG values ( $\mathrm{pH} 4.5$ and $\mathrm{pH} 7.0$ ) being $0 \mathrm{~kg} \mathrm{H}_{2} \mathrm{SO}_{4} / \mathrm{t}$. The AP calculated based on the total $\mathrm{S}$ and applying the factor of 31.25 was $9.56 \mathrm{~kg} \mathrm{CaCO}_{3} / \mathrm{t}$ (AP1) and $19.1 \mathrm{~kg}$ $\mathrm{CaCO}_{3} / \mathrm{t}$ with the factor of 62.5 (AP2). The minAP calculated based on minS was $1.31 \mathrm{~kg} \mathrm{CaCO}_{3} / \mathrm{t}$ applying the factor of 31.25 (minAP1) and $2.61 \mathrm{~kg} \mathrm{CaCO}_{3} / \mathrm{t}$ with the factor of 62.5 (minAP2). The NP according to the standard $\mathrm{ABA}$ test was $57.7 \mathrm{~kg} \mathrm{CaCO}_{3} / \mathrm{t}$, the carbNP calculated from the carbonate $\mathrm{C}$ concentration was $8.1 \mathrm{~kg} \mathrm{CaCO}_{3} / \mathrm{t}$ and the minNP calculated by mineralogy was $173 \mathrm{~kg} \mathrm{CaCO}_{3} / \mathrm{t}$. Based on the calculations, the main three minNP-contributing minerals were diopside $\left(148 \mathrm{~kg} \mathrm{CaCO}_{3} / \mathrm{t}\right)$, olivine $\left(12 \mathrm{~kg} \mathrm{CaCO}_{3} / \mathrm{t}\right)$ and serpentine $\left(6.49 \mathrm{~kg} \mathrm{CaCO}_{3} / \mathrm{t}\right)$. The Kevitsa waste rock material was classified as non-acid-generating by most of the applied ARD prediction methods, except when using the carbNP.

All samples contained some sulphides, which were mostly pyrrhotite and pyrite, with lesser amounts of chalcopyrite, pentlandite and sphalerite (Table 6). Most samples also contained some amounts of oxidised $\mathrm{Fe}$ sulphides transformed into sulphate-bearing $\mathrm{Fe}$ oxyhydroxides (or gypsum resulting from neutralisation), typically appearing around the healthy sulphides as a thin (a few micrometres) rim. The sulphides mainly appeared as single grains. The most common carbonates were calcite and dolomite, which were present in most carbonate-containing samples (Table 6). Magnesite was only present in both Horsmanaho samples and siderite in the Pampalo sample.

Silicates of intermediate-weathering intensity were the dominant type in the waste rock samples (Fig. 1). These minerals consist of $\mathrm{Mg}$ and Fe silicates and $\mathrm{Mg}$ or Fe-bearing aluminosilicates. Their content was greatest $(>40 \mathrm{wt} \%)$ in the samples Siilinjärvi old and Särkiniemi, respectively. The concentrations of the silicates of slow-weathering intensity were greatest (> $40 \mathrm{wt} \%$ ) in the samples Siilinjärvi new and Hällinmäki, respectively. All samples contained very slowly weathering silicates at contents below $20 \mathrm{wt} \%$, the highest amounts being detected in the samples Pampalo (19.7 wt\%) and Siilinjärvi new (15.6 wt\%).

The NAG pH was high $(\geq 7.5)$ and the NAG value correspondingly zero in the samples of group I and group II, excluding the sample Horsmanaho new, and in the samples Kevitsa and Laiva of group III (Table 7). Group III had dominantly low NAG pH values $(\leq 4.1)$. As presented in Fig. 2, the NAG pH was a pessimistic indicator in the cases of Horsmanaho new and Hällinmäki samples and over-optimistic in the case of group I samples.

A comparison of NP values obtained by different methods is presented in Fig. 3. The EN 15875 ABA test NP was highest in the Pampalo and Siilinjärvi new samples of group I, in the Kylylahti 2014 sample of group II and in the Hitura 2014, Hammaslahti and Laiva samples of group III. The mineralogical NP was highest in the Siilinjärvi old sample of group I, in both Horsmanaho samples of group II, and in the Särkiniemi, Kevitsa, Hitura 2016 and Hällinmäki samples of group III. The carbonate NP was usually the lowest of the different NP values, excluding the Pampalo sample of group I, in which minNP was lower, and group II samples, in which the ABA NP values of the both Horsmanaho samples were lower and the minNP of the Kylylahti 2014 sample was lower. In general, the smallest differences between NP values were observed in the group I samples (Fig. 3).

Based on the results presented in Table 8, carbonates are the main NP contributors in the group I samples of Pampalo (most NP from dolomite), Siilinjärvi old (dolomite) and Siilinjärvi new (calcite) and in the group II sample of Kylylahti 2014 (calcite). Silicate minerals dominate the NP potential of the other samples, the main NP contributor being biotite in Hitura 2014 and 2016, Hammaslahti, Särkiniemi and Laiva. Diopside was the most important NP mineral in the Kevitsa sample and aegerine-augite in the Hällinmäki sample.

Sulphur concentrations obtained using the Leco method were notably higher in the majority of the samples than the $\mathrm{S}$ concentrations calculated based on mineralogy (Table 7). The minS was higher in two samples, Kylylahti 2014 and Hitura 2014, which had the highest $\mathrm{S}$ concentrations in general (Leco S 4.32 and $3.35 \%$ and $\operatorname{minS} 4.97$ and $3.72 \%$, respectively). A comparison of AP values obtained using the EN 15875 ABA test and mineralogical calculations using the factor of 31.25 is presented in Fig. 4. EN ABA test AP1 values are higher in all samples, excluding the Kylylahti 2014 sample of group II. In general, the smallest differences between AP1 and minAP1 samples were observed in the group I samples (Fig. 4). 


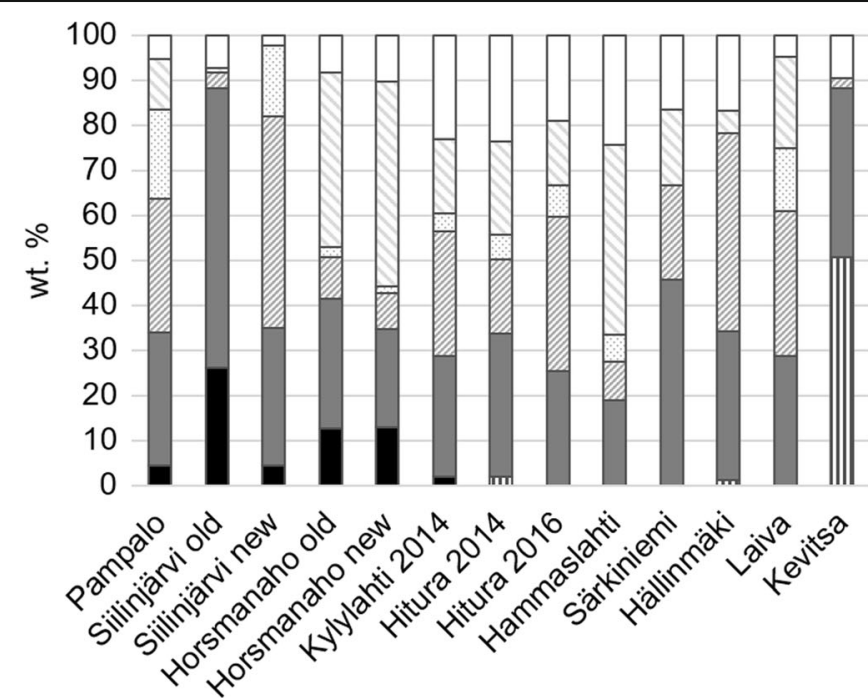

$\square$ Sulphides and unclassified minerals

口Inert silicates: quartz

$\square$ Very slow-weathering silicates

๒Slow-weathering

$\square$ Intermediate-weathering silicates

mFast-weathering silicates

- Carbonates

Fig. 1 Mineral groups according to weathering intensity after Sverdup (1990), including a group of sulphides and unclassified minerals

According to the ABA results (NP/AP ratios), samples of group I (Pampalo, Siilinjärvi old and new) consist of non-acid-generating waste rocks (Tables 9 and 10), whereas the other waste rocks (groups II and III) are acid-generating, except for the Kevitsa and Laiva waste rocks. Based on NAG pH and NAG values, the rock samples of the first group were non-acid-generating, similarly to the determination based on the ABA test. Congruently with the ABA test, the NAG test classified the Horsmanaho new sample from group II and the Hitura 2014 and Särkiniemi samples from group III as acid-generating. In contrast to the ABA test, the NAG test classified the Horsmanaho old and Kylylahti 2014 samples from group II and Kevitsa and Laiva samples from group III as non-acid-generating.

\section{Discussion}

This study focused on 14 waste rock and 14 corresponding seepage water samples from 10 Finnish mine sites where thorough static testing was undertaken. The investigated static tests were compared with each other and with the corresponding seepage pHs. All the static tests have known limitations related, for instance, to the rock texture, mineral surface area, mineral assemblage

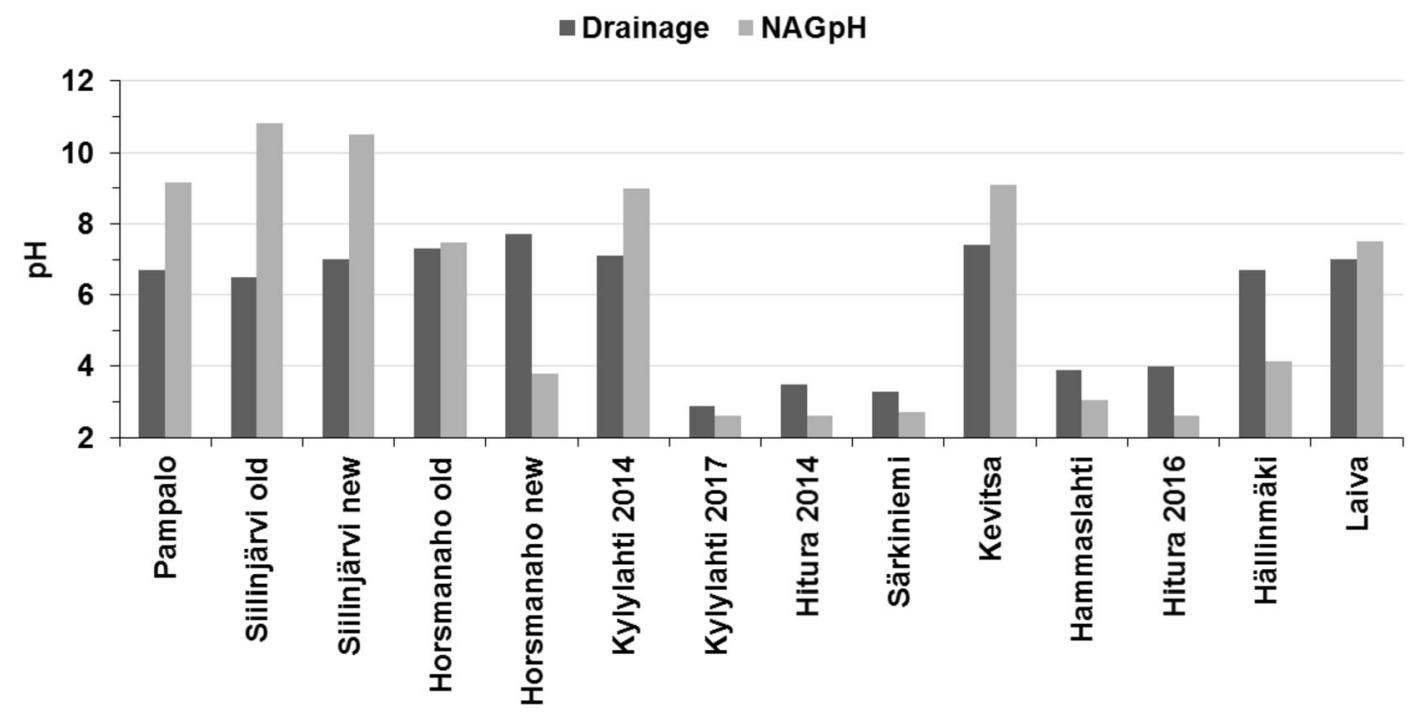

Fig. 2 Comparison of the NAG $\mathrm{pH}$ and drainage $\mathrm{pH}$ values 


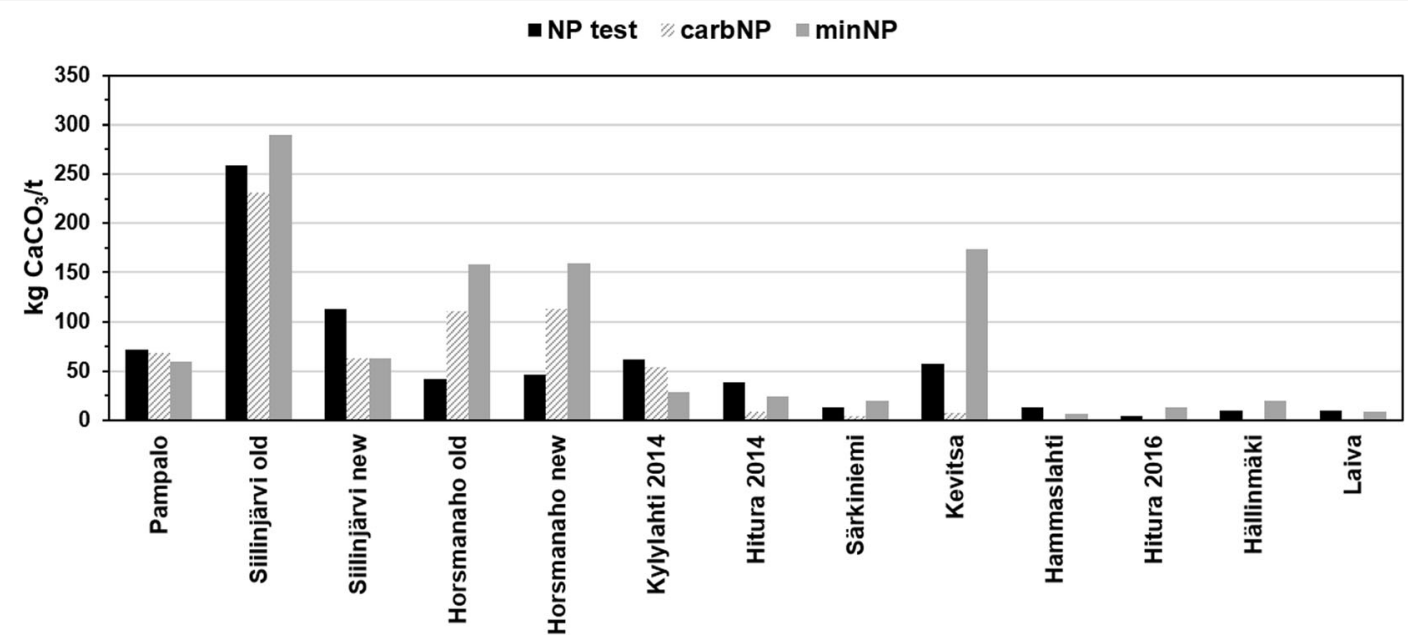

Fig. 3 Comparison of NP values obtained using different methods

and mineral impurities that might affect the weathering rate, small sample sizes and crushing of the samples (White III et al. 1999; Paktunc 1999b; Jambor 2003; Parbhakar-Fox and Lottermoser 2015; Dold 2017). Compared to this study, in which pulverised rock samples were used and valuable information on the rock texture was mainly lost, the mineralogical prediction accuracy can be further enhanced with a textural investigation and assessment of the intact rocks (ParbhakarFox et al. 2011; Brough et al. 2013). For this purpose, the acid rock drainage index (ARDI) has been developed, as part of the geochemistry-mineralogy-texture (GMT) approach (Parbhakar-Fox et al. 2011).

For both the commonly used static tests and the calculations based on mineralogy, the key element in successful characterisation is representative sampling (Price 2009). For this study, thorough sampling of the waste rock piles was not possible and only composite surface samples were collected. The known limitations should be taken into account when inspecting the geochemical rock analysis results and drainage quality data. Correct environmental sampling techniques are presented, for example, by McLemore et al. (2014).

It should be emphasized that while the static tests and mineralogical calculations are suitable for preliminary screening in the ARD assessment of mine waste, more detailed and site-specific investigations should still be carried out, not to exclude investigations to predict metal-rich neutral mine drainage (NMD, Dold 2017). As this study concentrated on preliminary screening

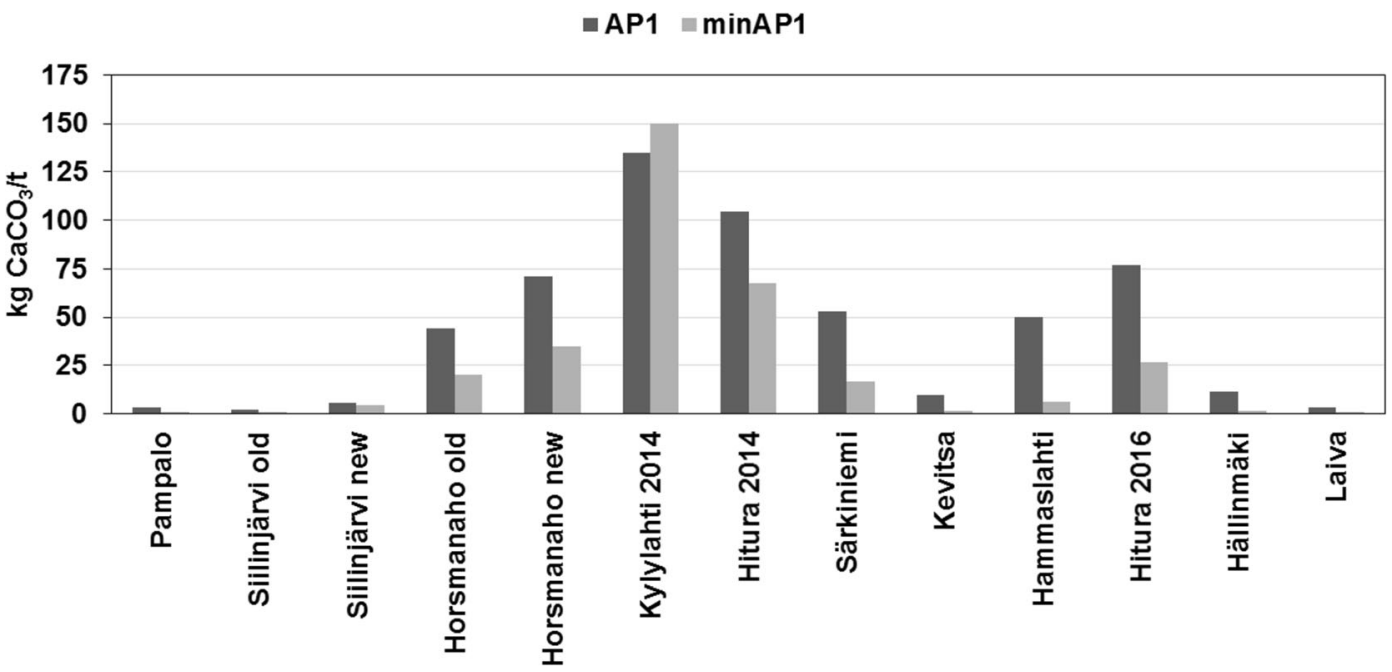

Fig. 4 Comparison of AP values obtained using the EN 15875 ABA test and mineralogical calculation 
purposes, assuming sulphide oxidation by oxygen, more complicated further reactions such as oxidation by $\mathrm{Fe}^{3+}$ (Dold 2010) and the precipitation of $\mathrm{Fe}^{3+}$ and $\mathrm{Al}^{3+}$ hydroxides (Stumm and Morgan 1996; Lottermoser 2010) should be taken into account when performing detailed site-specific investigations.

According to the results, ARD prediction based on SEM mineralogy and mineralogical NP calculation appears to be an accurate tool compared to the common static laboratory methods. The mineralogical ARD calculation could, for instance, be incorporated in mineralogical analyses and the results regarded to be as adequate as the results from the commonly used static tests. The weathering groups of minerals according to Sverdrup (1990) might be considered to be too broad for ARD prediction. For example, pyroxene, amphibole, biotite and chlorite are all grouped as intermediateweathering with the same relative reactivity value (Jambor and Blowes 1998). Nevertheless, mineralogical investigation reveals information about the actual minerals corresponding to the AP and NP, which, besides the calculation of AP and NP values, also permits the assessment of reaction rates of significant minerals compared to each other. This study highlighted the need for further investigation into the contribution of silicates to the neutralisation capacity in calcite-poor environments and especially into the ability of silicates to react to potentially rapid acid surges generated by pyrrhotite weathering.

\section{Determination of AP}

The Fe sulphides pyrrhotite and pyrite, respectively, appear to be the most widely occurring sulphide species in Finnish waste rocks, also being responsible for the majority of acid production. Pyrrhotite was calculated to be the main AP-producing mineral at $6 / 10$ of the mine sites. This is contradictory to the common assumption that pyrite is the greatest contributor to ARD (Nordstrom and Alpers 1999). As the common static ABA tests do not provide information on the S minerals, as pointed out by Dold (2017), the oxidation of different sulphides produces fewer protons than pyrite, and the main problem of the EN 15875 ABA tests seems to be the overestimation of the AP. Besides the different numbers of protons released, sulphide species also have different resistances to oxidation. According to Jambor (1994), the oxidation resistance from least to highest is pyrrhotite $\rightarrow$ sphalerite/galena $\rightarrow$ pyrite-arsenopyrite $\rightarrow$ chalcopyrite $\rightarrow$ magnetite. Thus, as pointed out by Jambor and Blowes (1998), although pyrite can theoretically generate more acid than pyrrhotite, the slower weathering rate of pyrite, and hence the lower capacity to overwhelm neutralisation reactions, introduces uncertainties in predictions based on static ABA tests. The oxidation resistance order of different sulphide species might change when factors such as biological (Kuyucak 2002) and galvanic (Kwong et al. 2003) interactions are considered. The stability of individual sulphides is also related to the interstitial impurities and trace elements, which usually render sulphides more susceptible to weathering (Kwong 1993).

As pyrrhotite appears to be the most important acid producer at Finnish mine sites, more effort should be directed to studies related to the occurrence of different pyrrhotite types and the ability of silicates to resist a possible rapid pyrrhotite-originated acid surge. The reaction rates of pyrrhotite have been noted to be up to 100 times faster than those of pyrite at $25^{\circ} \mathrm{C}$ and in atmospheric oxygen (Nicholson 1994; Nicholson and Scharer 1994). The main factor for higher oxidation rate is the higher surface area of pyrrhotite compared with the other sulphides, e.g. pyrite (Janzen et al. 2000). Although in general the oxidation of pyrrhotite is relatively fast, the structural differences can cause variable dissolution behaviour of pyrrhotite (Thomas et al. 1998).

When inspecting uncertain cases, e.g. samples from Hällinmäki and Särkiniemi, the uncertainties related to ARD prediction by static methods could be reduced by examining the sulphide species. In many Finnish waste rock facilities, the neutralisation capacity is related to silicate minerals, and fast-reacting carbonates are absent. If the main AP contributor in the Nordic climate is slowly weathering sulphide, such as chalcopyrite in Hällinmäki, this suggests that the silicate-based NP could respond to the acidity, as also appears to be the case when observing the actual drainage quality at the Hällinmäki mine site. On the other hand, if the main AP contributor is fast-oxidising pyrrhotite, it can produce a surge of acidity that will overwhelm the immediately available neutralising capacity of associated silicates, as can be observed at the Särkiniemi mine site.

The AP is often calculated based on the total $\mathrm{S}$ concentration, which has been debated by White et al. (1999) and Parbhakar-Fox et al. (2011), among others. The main complication is related to the fact that nonacid-forming S-bearing minerals, such as gypsum and 
barite, are included in the analysis and therefore in the AP potential. According to the mineralogical investigations in this study, barite does not appear to be a very common mineral in Finnish waste rock material, although some well-known exceptions exist, e.g. the barite-bearing Pyhäsalmi (Mäki et al. 2015) and Pahtavaara (Korkiakoski and Kilpelä 1997) deposits. Small amounts of gypsum and silicate-gypsum mixture were detected at 5/10 mine sites. The detected amounts were small, between 0.01 and $0.02 \mathrm{wt} \%$, but considering that some gypsum probably remained undetected, gypsum might be a critical contributor to the total $S$ and therefore the AP, increasing uncertainty in some ARD assessment cases. In these cases, pre-treating samples to remove gypsum should be considered. For example, samples can be digested with sodium carbonate to remove sulphate minerals (Lapakko 2002).

As the AP values obtained for static ABA testing are usually already overestimated, as discussed above, the use of the factor 62.5 instead of the commonly used 31.25 results in even more pessimistic ARD predictions (Tables 9 and 10). On the other hand, the use of the higher factor is well justified in cases when bicarbonate is the main product of carbonate dissolution (Dold 2017). In addition, the ARD predictions based on mineralogically calculated minNP and minAP obtained with the factor of 62.5 (Table 10) appear to result in realistic predictions compared to the actual drainage qualities.

\section{Determination of the NP}

According to the calculated NP values, silicates appear to be an important contributor to ARD prevention at Finnish mine sites. Carbonates are the main source of NP at $4 / 10$ of the investigated mine sites, of which calcite is the main source of NP at only two sites. At the remaining $6 / 10$ of the investigated mine sites, silicate minerals are the most important contributors to the NP. Of the silicate minerals, biotite was observed to be the most important contributor to the neutralisation capacity at $4 / 10$ of the investigated mine sites, followed by the other silicate minerals of the fast- and intermediateweathering groups. Chlorite and mica have also been demonstrated to have a long-term acid-neutralising capacity in previous studies (Becker et al. 2015). As carbonates appear to be less common in Finnish mine wastes, the application of carbNP (NP calculated from the carbonate $\mathrm{C}$ concentration) is not widely recommendable. On the other hand, carbNP can be used in
ARD predictions for mine wastes in which carbonates are slowly weatherable (e.g. magnesite) and therefore weakly leachable in the NP test and in which carbonates are main contributors to the neutralisation capacity.

Differences between the NP measured using the standard EN 15875 method and minNP calculated from the mineral content can be observed in Fig. 5. Magnesite appears to be considerably underestimated in the measured NP. According to the dissolution studies by Hoşgün and Kurama (2012), magnesite should mostly dissolve in $1 \mathrm{M} \mathrm{HCl}$ solution. On the other hand, the results of this study suggest that most of the magnesite did not dissolve in the $1 \mathrm{M} \mathrm{HCl}$ used in the NP measurement, and magnesite has also been noted to react slowly in laboratory ABA tests by Paktunc (1999b). Based on Fig. 5, other minerals that might be undervalued by the laboratory measured NP (i.e. not well leached in $\mathrm{HCl}$ ) are diopside, aegerine-augite, hornblende, anorthite and biotite. In some cases, the higher minNP values could also result from the fact that the mineralogical calculations were based on the relative reactivity values determined in slightly acid conditions (pH 5.0; Sverdrup 1990), whereas in most of the investigated mine waste sites, the $\mathrm{pH}$ is close to neutral. Therefore, the minNP values might overestimate the neutralising capacity, as the overall mineral dissolution is lower at a higher pH (Heikkinen and Räisänen 2008).

Apparently, the measured NP might slightly overestimate the neutralisation capacity of olivine, which has also been noted by Heikkinen and Räisänen (2008). This might be due to the $\mathrm{HCl}$ used in the EN ABA test, which dissolves some silicates more efficiently than is assumed in minNP calculations (Heikkinen and Räisänen 2008). Acids have been noted to more readily dissolve olivine compared to various other minerals, e.g. pyroxenes and some phyllosilicates (Terry 1983). Underestimation of biotite (due to its abundance) in the NP based on titration can also be noted in the Hitura 2016 sample.

The behaviour and dissolution of minerals in static laboratory tests and field sites are complicated and affected by several factors (Mase 1961; Prosser 1970; Currel et al. 1972; Sanemasa et al. 1972; Terry 1983; Nagy 1995; Critelli et al. 2014). The dissolution of significant NP-contributing minerals should be more thoroughly investigated, especially regarding common ARD prediction methods, as according to the results, the EN ABA test and NAG test do not correctly consider certain mineral types. In general, as silicate minerals 
appear to have significant importance for the neutralisation capacity and therefore for ARD prediction, the relative reactivities and dissolution rates of these minerals should be more thoroughly investigated in acids and mine waste environments and taken into greater account in NP assessments.

\section{ARD prediction based on the NAG test}

When using the traditional $\mathrm{NAG} \mathrm{pH}$ criterion of $\mathrm{pH} \leq 4.5$ for potentially acid-generating rock material, the NAG test $\mathrm{pH}$ was observed to be a pessimistic indicator in some cases. In the Horsmanaho new sample, the main AP contributor was a high amount (3.59 wt\%) of fastreacting pyrrhotite, whereas the main NP contributor was magnesite, which has also been noted to react slowly in laboratory ABA tests by Paktunc (1999b). The relatively low NAG pH of the Hällinmäki sample suggests that otherwise slowly weathering chalcopyrite is oxidised by the hydrogen peroxide, whereas the neutralisation capacity of the silicates could not be used. If the NAG pH, with the criterion of a pH between 3.21 and 4.52, was used for the uncertainty zone (UC), as proposed by $\mathrm{Oh}$ et al. (2017), Horsmanaho new and Hällinmäki samples would be classified as UC instead of PAF. It should be noted that when the NAG pH is lower than 4.5, the short-term risk of acid release is high, as a less acid-neutralising capacity might be readily available (Oh et al. 2017).

The results suggest that the weathering state of the sample has a significant impact on the NAG test performance. As was suspected based on the AMIRA guidebook (Smart et al. 2002), the most basic single-addition
NAG test was not sufficient for the Kylylahti 2014 sample, which contained a high amount $(9.53 \mathrm{wt} \%)$ of sulphides. Apparently, the added hydrogen peroxide was depleted before all the reactive sulphides were oxidised, as incomplete oxidation of sulphides can already appear in samples containing $<0.7-1 \mathrm{wt} \%$ pyritic sulphide (Stewart 2005). For samples containing high amounts of sulphides, the AMIRA guidebook (Smart et al. 2002) recommends the use of sequential NAG tests, where successive additions of peroxide are used. The Kylylahti 2014 sample was also relatively unweathered and fresh, which might affect the efficiency of hydrogen peroxide. The singleaddition NAG test of the Kylylahti 2017 rock sample, which was similar to the Kylylahti 2014 sample but more weathered, resulted in more realistic NAG values.

The chemical mechanisms that control the stability and behaviour of the hydrogen peroxide solution are still insufficiently understood (Charles et al. 2015). Therefore, further investigation of the NAG test's suitability for different rock types is needed. For example, the slowly reacting buffering minerals might require a longer time to react with hydrogen peroxide than overnight, which is specified in the AMIRA guidebook (Smart et al. 2002). Nevertheless, the NAG pH can be considered as a plausible single indicator in ARD assessment, as the measurement is simple and applicable to various samples (Oh et al. 2017). To enhance the usability of the NAG test, Parbhakar-Fox et al. (2018) highlight the importance of understanding the mineralogy of the sample prior to testing and developing a site-specific NAG testing protocol prior to new extractive waste classification projects. Furthermore, to reduce the discrepancies
Fig. 5 Comparison of the mineralogical NP with the measured NP values and the three most significant minNPcontributing minerals and percentage contribution of the minerals to the total calculated minNP. Abbreviations for the minerals: act $=$ actinolite, aeg $=$ aegerine-augite, alm = almandine, an $=$ anorthite, $\mathrm{bt}=$ biotite, $\mathrm{cc}=$ calcite, chl $=$ chlorite, dip = diopside, do $=$ dolomite, fhbl $=$ Fe-hornblende, mgs = magnesite, $\mathrm{mhbl}=\mathrm{Mg}$-hornblende, $\mathrm{ol}=$ olivine, $\mathrm{phl}=$ phlogopite, $\operatorname{srp}=$ serpentine, tlc $=$ talc

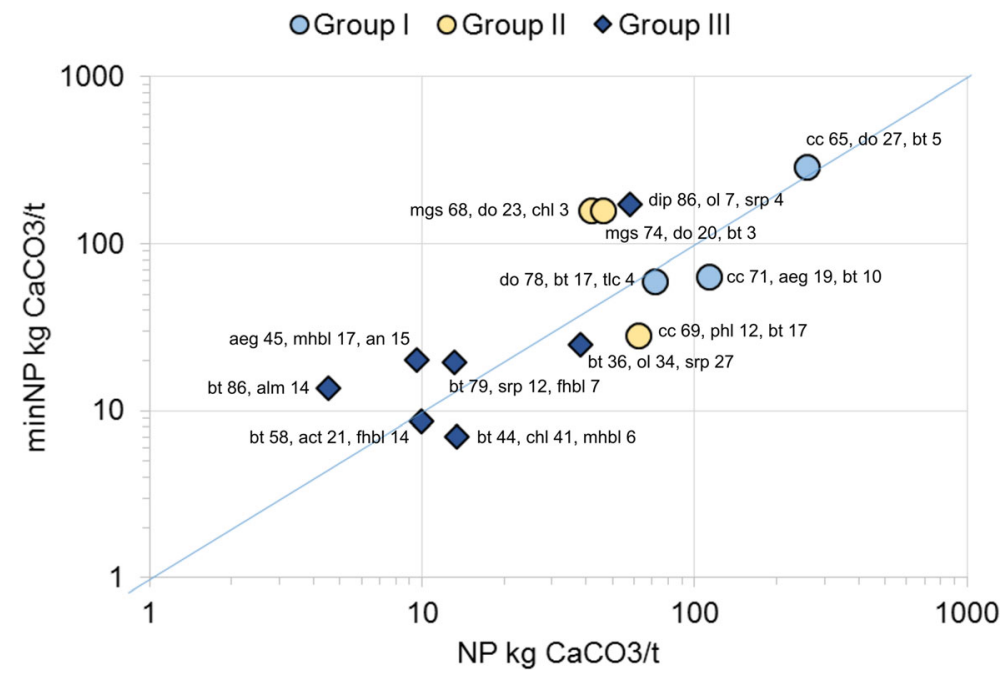


between laboratory results, standard experiment protocols and standard reference materials should be developed and used (Parbhakar-Fox et al. 2018).

\section{Conclusions}

At Finnish mine waste sites, pyrrhotite appears to more commonly occur than pyrite. Pyrrhotite is also responsible for the main part of acid production. The standard ABA test results in too pessimistic ARD predictions, as the AP is overestimated by assuming that all $\mathrm{S}$ is pyritic.

At most of the investigated mine sites, silicate minerals were found to be the most important contributors to the neutralisation potential. Of the silicate minerals, biotite was observed to be the most important contributor to the total NP, followed by the other silicate minerals of the fast- and intermediate-weathering groups. As the carbonates appear to be less important NP producers in Finnish mine wastes, the application of carbNP is not widely recommendable. For the NAG test, the slowly reacting buffering minerals might require a longer time to react with hydrogen peroxide than overnight, which is specified in the AMIRA guidebook.

As silicate minerals appear to have significant importance for the neutralisation capacity and therefore ARD prediction at Finnish mine waste sites, the behaviour, relative reactivities and dissolution rates of these minerals should be more thoroughly investigated. More effort should especially be directed to studies related to the ability of silicates to resist the potentially rapid acid surges originating from pyrrhotite oxidation.

SEM mineralogy-based AP and NP calculation appears to be an efficient tool for ARD prediction. As an enhancement to the common static laboratory tests, mineralogical investigation reveals the actual minerals corresponding to the AP and NP, also permitting assessment of the reaction rates of significant minerals compared to each other. If sufficient suitable mineralogical data are available, there is less need to perform static ARD tests, which reduces analytical costs. Instead, funding could be used to investigate the possibility of NMD or the mobility of potentially harmful elements, as well as for more detailed kinetic testing of waste materials.

Acknowledgements The data for this study were obtained as part of the Closedure project (2012-2015), funded by the TEKES Green Mining Programme and the KaiHaMe project (2015-2018), funded by the European Regional Development Fund. The authors would like to thank the mining companies for participating in these projects and for facilitating the necessary fieldwork.

Open Access This article is distributed under the terms of the Creative Commons Attribution 4.0 International License (http:// creativecommons.org/licenses/by/4.0/), which permits unrestricted use, distribution, and reproduction in any medium, provided you give appropriate credit to the original author(s) and the source, provide a link to the Creative Commons license, and indicate if changes were made.

Publisher's Note Springer Nature remains neutral with regard to jurisdictional claims in published maps and institutional affiliations.

\section{References}

Appelo, C. A. J., \& Postma, D. (2010). Geochemistry, groundwater and pollution (2nd ed.). Leiden: CRC, Balkema.

Becker, M., Dyantyi, N., Broadhurst, J. L., Harrison, S. T. L., \& Franzidis, J.-P. (2015). A mineralogical approach to evaluating laboratory scale acid rock drainage characterisation tests. Minerals Engineering, 80, 33-36.

Blowes, D. W., \& Jambor, J. L. (1990). The pore-water geochemistry and the mineralogy of the vadose zone of sulfide tailings, Waite amulet, Quebec, Canada. Applied Geochemistry, 5, 327-346.

Blowes, D. W., \& Ptacek, C. J. (1994). Acid-neutralization mechanisms in inactive mine tailings. In J. L. Jambor, \& Blowes, D. W. (Eds.), The environmental geochemistry of sulfide mine wastes. Mineralogical Association of Canada, short course series, volume 31, (pp. 95-116).

Blowes, D. W., Ptacek, C. J., Jambor, J. L., Weisener, C. G., Paktunc, D., Gould, W. D., \& Johnson, D. B. (2014). 11.5 - the geochemistry of acid mine drainage. In H. D. Holland \& K. K. Turekian (Eds.), Treatise on geochemistry (second edition) (pp. 131-190, ISBN 9780080983004). Oxford: Elsevier. https://doi. org/10.1016/B978-0-08-095975-7.00905-0.

Brough, C. P., Warrender, R., Bowell, R. J., Barnes, A., \& Parbhakar-Fox, A. (2013). The process mineralogy of mine wastes. Minerals Engineering, 52, 125-135.

Charles, J., Barnes, A., Declercq, J., Warrender, R., Brough, C., \& Bowell, R. (2015). Difficulties of interpretation of NAG test results on net neutralizing mine wastes: Initial observations of elevated $\mathrm{pH}$ conditions and theory of $\mathrm{CO} 2$ disequilibrium. In Proceedings of the 10th international conference on acid rock drainage and IMWA annual conference, April 21-24. Santiago, Chile: Gecamin.

Chen, Y., \& Brantley, S. L. (1998). Diopside and anthophyllite dissolution at $25^{\circ}$ and $90^{\circ} \mathrm{C}$ and acid pH. Chemical Geology, 147, 233-248.

Colmer, A. R., \& Hinkle, M. E. (1947). The role of microorganisms in acid mine drainage. Science, 106, 253-256.

Critelli, T., Marini, L., Schott, J., Mavromatis, V., Apollaro, C., Rinder, T., De Rosa, R., \& Oelkers, E. H. (2014). Dissolution rates of actinolite and chlorite from a wholerock experimental study of metabasalt dissolution from 2 $\leq \mathrm{pH} \leq 12$ at $25^{\circ} \mathrm{C}$. Chemical Geology, 390, 100-108. 
Currell, B. R., Midgley, H. G., \& Seaborne, M. A. (1972). Formation of poly-organosiloxanes from silicate minerals. Nature Physical Science, 236, 108.

Dold, B. (2010). Basic concepts in environmental geochemistry of sulfidic mine-waste management. In S. E. Kumar (Ed.), Waste Management, ISBN 978-953-7619-84-8.

Dold, B. (2014). Evolution of acid mine drainage formation in sulphidic mine tailings. Minerals, 4(2), 621-641.

Dold, B. (2017). Acid rock drainage prediction: A critical review. Journal of Geochemical Exploration, 172, 120-132.

EC (2009). COMMISSION DECISION of 30 April 2009 completing the technical requirements for waste characterisation laid down by Directive 2006/21/EC of the European Parliament and of the Council on the management of waste from extractive industries (2009/360/EC).

European Committee for standardization (2008). prEN 15875, Characterization of waste - Static test for the determination of acid potential and neutralization potential of sulfidic waste.

Finnish Government Decree 190/2013 (2013). Government Decree on Mining Wastes 14.3.2013. (In Finnish or Swedish).

GTK (2017a). Mineral deposit report 284: Pampalo. In Geological Survey of Finland, Mineral deposits-report database. http://tupa.gtk.fi/karttasovellus/mdae/raportti/284_Pampalo. pdf. Accessed 22 January 2018.

GTK (2017b). Mineral deposit report 103: Virtasalmi. In Geological Survey of Finland, Mineral deposits-report database. http://tupa.gtk.fi/karttasovellus/mdae/raportti/103 Virtasalmi.pdf. Accessed 22 January 2018.

Hämäläinen, I. (1987). Hammaslahden sinkkimalmi. Julkaisematon Pro gradu-tutkielma. In Turun yliopisto, Geologian ja mineralogian laitos. 110 p. (In Finnish).

Haney, E. B., Haney, R. L., Hossner, L. R., \& White, G. N. (2006). Neutralization potential determination of siderite (FeCO3) using selected oxidants. Journal of Environmental Quality, 35(3), 871-879.

Heikkinen, P. M., \& Räisänen, M. L. (2008). Mineralogical and geochemical alteration of Hitura sulphide mine tailings with emphasis on nickel mobility and retention. Journal of Geochemical Exploration, 97, 1-20.

Hoşgün, H., \& Kurama, H. (2012). Dissolution kinetics of magnesite waste in $\mathrm{HCl}$ solution. Industrial and Engineering Chemistry Research, 51(3), 1087-1092.

Hyvärinen, L. (1969). On the geology of the copper ore field in the Virtasalmi area, eastern Finland. Bulletin de la Commission Géologique de Finlande, 240, 82.

Janzen, M. P., Nicholson, R. V. \& Scharer, J. M. (2000). Pyrrhotite reaction kinetics: reaction rates for oxidation by oxygen, ferric iron, and for nonoxidative dissolution. Geochimica et Cosmochimica Acta 64 (9), 1511-1522.

Jambor, J. L. (1994). Mineralogy of sulfide-rich tailings and their oxidation products. In D. W. Blowes \& J. L. Jambor (Eds.), The Environmental Geochemistry of Sulfide Mine-Wastes, Short Course Handbook (Vol. 22, pp. 59-102). Waterloo, Ontario, Canada: Mineralogical Association of Canada.

Jambor, J. L. (2003). Mine-waste mineralogy and mineralogical perspectives of acid-base accounting. In J. L. Jambor, D. W. Blowes, \& A. I. M. Ritchie (Eds.), Environmental aspects of mine wastes, Mineralogical Association of Canada (Vol. 31, pp. 117-145). Ottawa, Ontario, Canada, short course series.

Jambor, J. L., \& Blowes, D. W. (1998). Theory and applications of mineralogy in environmental studies of sulfide-bearing mine wastes. In L. J. Cabri \& D. J. Vaughan (Eds.), Modern approaches to ore and environmental mineralogy (Vol. 27, pp. 367-401). Ottawa, Ontario, Canada, short course series: Mineralogical Association of Canada.

Jamieson, H. E., Walker, S. R., \& Parsons, M. B. (2015). Mineralogical characterization of mine waste. Applied Geochemistry, 57, 85-105.

Karppanen, T. (1986). The Hammaslahti mine. In: K. Kojonen (Ed.), Prospecting in areas of glaciated terrain 1986: Guide to the field excursion for the symposium in eastern Finland, September 3-5 1986 (pp. 34 40).

Kontinen, A., Peltonen, P., \& Huhma, H. (2006). Description and genetic modelling of the Outokumpu-type rock assemblage and assiciated sulphide deposits. Geological Survey of Finland, Archive Report M 10.4/2006/1, 380 p.

Kontoniemi, O., \& Forss, H. (1997). Tutkimustyöselostus Leppävirran kunnassa valtausalueilla Särki 1 (kaiv. rek. nro 5422/1) ja Särki 2 (kaiv. rek. nro 5422/2) suoritetuista nikkelimalmitutkimuksista vuosina 1993-1996. Geological Survey of Finland, Archive report, M06/3241/-97/2/10. (In Finnish), 14 p.

Korkiakoski, E., \& Kilpelä, M. (1997). The Komatite-hosted Pahtavaara gold mine near Sodankylä, northern Finland. In E. Korkiakoski, \& Sorjonen-Ward, P. (Eds.), Ore deposits of Lapland in northern Finland and Sweden. Research and exploration - Where do they meet? 4th biennial SGA Meeting, August 11-13, 1997, Turku, Finland, excursion guidebook B1. Geological Survey of Finland, Guide 43, (pp. 27-29).

Kuronen, E., \& Tuokko, I. (1997). Horsmanaho Talc Mine. In K. Loukola-Ruskeeniemi, \& Sorjonen-Ward, P. (Eds.), Excursion Guidebook A4 - Ore Deposits in Eastern Finland. 4th Biennial SGA Meeting, August 11-13, 1997, Turku, Finland. Geological Survey of Finland, Guide 42, pp. 39-42.

Kuyucak, N. (2002). Role of microorganisms in mining: Generation of acid rock drainage and its mitigation and treatment. The European Journal of Mineral Processing and Environmental Protection, 2(3), 179-196.

Kwong, Y. T. (1993). Prediction and prevention of acid rock drainage from a geological and mineralogical perspective. MEND, $47 \mathrm{p}$.

Kwong, Y. T., Swerhone, G. W., \& Lawrence, J. R. (2003). Galvanic sulphide oxidation as a metal-leaching mechanism and its environmental implications. Geochemistry: Exploration, Environment, Analysis, 3, 337-343.

Lapakko, K. (2002). Metal mine rock and waste characterization tools: An overview. Mining, Minerals and Sustainable Development (MMSD) working paper 67, $31 \mathrm{p}$.

Lawrence, R. W., \& Scheske, M. (1997). A method to calculate the neutralization potential of mining wastes. Environmental Geology, 32, 100-104.

Lawrence, R. W., \& Wang, Y. (1997). Determination of neutralization potential in the prediction of acid rock drainage, proc. 4th international conference on acid rock drainage, Vancouver, BC, (pp. 449-464).

Lawrence, R. W., Poling, G. W., Ritcey, G. M., \& Marchant, P. B. (1989). Assessment of predictive methods for the determination of AMD potential in mine tailings and waste rock, tailings and effluent management (pp. 317-331). New York: Pergamon Press. 
Lawrie, K. C. (1992). Geochemical characterisation of a polyphase deformed, altered and high grade metamorphosed volcanic terrane: Implications for the tectonic setting of the Svecofennides, south-Central Finland. Precambrian Research, 59, 171-205.

Lottermoser, B. G. (2010). Mine wastes: Characterization, treatment and environmental impacts (3rd ed.). Berlin: SpringerVerlag $400 \mathrm{p}$.

Loukola-Ruskeeniemi, K., Gaal, G., \& Karppanen, T. (1993). Geochemical and structural characteristics of a sedimenthosted copper deposit at Hammaslahti: Comparison with Besshi-type massive sulphide deposits. In: K. LoukolaRuskeeniemi (Ed.), Geochemistry of Proterozoic metamorphosed black shales in eastern Finland, with implications for exploration and environmental studies. Academic dissertation, University of Helsinki, Finland. Paper III.

Mäki, T., Imaña, M., Kousa, J. \& Luukas, J. (2015). The VihantiPyhäsalmi VMS Belt. In: W. Maier, Lahtinen, R., \& O'brien, H. (Eds.), Mineral deposits of Finland, Chapter 7, (pp. 507-530).

Makkonen, H. (2012). Hitura Ni. In P. Eilu, Ahtola, T., Äikäs, O., Halkoaho, T., Heikura, P., Hulkki, H., Iljina, M., Juopperi, H., Karinen, T., Kärkkäinen, N., Konnunaho, J., Kontinen, A., Kontoniemi, O., Korkiakoski, E., Korsakova, M., Kuivasaari, T., Kyläkoski, M., Makkonen, H., Niiranen, T., Nikander, J., Nykänen, V,. Perdahl, J.-A., Pohjolainen, E., Räsänen, J., Sorjonen-Ward, P., Tiainen, M., Tontti, M., Torppa, A., \& Västi, K. (Eds), Metallogenic areas in Finland, Geological Survey of Finland, Special Paper 53, pp. 207-342, 90 figures and 43 tables.

Makkonen, H., \& Halkoaho, T. (2007). Whole rock analytical data (XRF, REE, PGE) for several Svecofennian (1.9 Ga) and Archean (2.8 Ga) nickel deposits in eastern Finland. Geological Survey of Finland, Archive report, M19/3241/ 2007/32, 49 p.

Mase, H. (1961). On the reactivity of various silicates towards acids. Bulletin of the Chemical Society of Japan, 34, 214-225.

McLemore, V., Smith, K. \& Russell, C. (2014). Sampling and monitoring for the mine life cycle. In Management Technologies for Metal Mining Influenced Water Series, v. 6. Society for Mining. Metallurgy, and exploration, Inc., $191 \mathrm{p}$.

Mehta, N., Dino, G. A., Ajmone, M. F., Lasagna, M., Romè, C., \& De Luca, D. A. (2018). Extractive waste management: A risk analysis approach. Science of the Total Environment, 622623, 900-912.

MEND (1991). Acid rock drainage prediction manual. In Canmet - MSL division, Department of Energy, mines and resources. Canada. MEND project 1.16.1b, $77 \mathrm{p}$.

Meriläinen, M., Ekberg, M., Lovén, P., Koivistoinen, P., Hakola, J., \& Strauss, T. (2008). Updated Reserve and Resource Estimate of the Hitura Nickel Mine in Central Finland. In Belvedere Resources. National Instrument 43-101 Technical Report, $65 \mathrm{p}$.

Miller, S., \& Jeffery, J. (1995). Advances in the prediction of acid generating mine waste materials. In N. J. Grundon \& L. C. Bell (Eds.), Proceedings of the second Australian acid mine drainage workshop (charters towers, Queensland, 28-31 march 1995) (pp. 33-43). Brisbane: Australian Centre for Minesite Rehabilitation Research.

Miller, S., Robertson, A., \& Donahue, T. (1997). Advances in acid drainage prediction using the net acid generating (NAG) test. In Proceedings fourth international conference on acid rock drainage, (pp. 533-547). Vancouver, B. C. Canada May 31 June 6, 1997, volume II.

Mineralogy Database (2017). Mineralogical data. retrieved August 31, 2017, from http://webmineral.com/

Morin, K. A., \& Hutt, N. M. (1999). Internet case study \#10: Comparison of NAG results to ABA results for the prediction of acidic drainage. Minesite Drainage Assessment Group (MDAG). http://www.mdag.com/case_studies/cs1-99.html. Accessed 15 January 2018.

Nagy, K L. (1995). Dissolution and precipitation kinetics of sheet silicates. In A. F. White \& S. L. Brantley (Eds.), Chemical weathering rates of silicate minerals. Reviews in Mineralogy $31,583 p$.

Nicholson, R. V. (1994). Iron-sulfide oxidation mechanisms: Laboratory studies. In J. L. Jambor \& D. W. Blowes (Eds.), Environmental geochemistry of sulfide mine-wastes (Vol. 22, pp. 163-183). Canada, short course series: Mineralogical Association of Canada.

Nicholson, R. V., \& Scharer, J. M. (1994). Pyrrhotite oxidation kinetics. In C. N. Alpers \& D. W. Blowes (Eds.), Environmental geochemistry of sulfide oxidation (Vol. 550, pp. 14-30). Washington, D.C.: American Chemical Society.

Nordstrom, D. K. (2000). Advances in the hydrogeochemistry and microbiology of acid mine waters. International Geology Review, 42(6), 499-515. https://doi.org/10.1080 100206810009465095 .

Nordstrom, D. K., \& Alpers, C. N. (1999). Geochemistry of acid mine waters. In G. S. Plumlee \& M. J. Logsdon (Eds.), The Environmental Geochemistry of Mineral Deposits, Part A: Processes, Techniques and Health Issues, Reviews in Economic Geology (Vol. 6A, pp. 133-160). Littleton: Society of Economic Geologists.

O'Brien, H., Heilimo, E. \& Heino, P. (2015). The Archean Siilinjärvi carbonatite complex. In: W. Maier, Lahtinen, R. \& O'Brien, H. (Eds.), Mineral deposits of Finland. Elsevier. ISBN: 978-0-12-410438-9.

Oh, C., Ji, S., Yim, G., \& Cheong, Y. (2017). Evaluation of net acid generation $\mathrm{pH}$ as a single indicator for acid forming potential of rocks using geochemical properties. Environmental Monitoring and Assessment, 189, 165.

Paktunc, A. D. (1999a). Discussion of "a method to calculate the neutralization potential of mining wastes" by Lawrence and Scheske. Environmental Geology, 38(1), 82-84.

Paktunc, A. D. (1999b). Mineralogical constrains on the determination of neutralization potential and prediction of acid mine drainage. Environmental Geology, 39(2), 103-112.

Papunen, H. (1986). Suomen metalliset malmiesiintymät. In H. Papunen, I. Haapala, \& P. Rouhunkoski (Eds.), Suomen malmigeologia. Metalliset malmiesiintymät (pp. 133-214). Mänttä: Suomen geologinen seura ry. Mäntä Kirjapaino Oy (in Finnish).

Parbhakar-Fox, A., \& Lottermoser, B. G. (2015). A critical review of acid rock drainage prediction methods and practices. Minerals Engineering, 82, 107-124.

Parbhakar-Fox, A., Edraki, M., Walters, S., \& Bradshaw, D. (2011). Development of a textural index for the prediction of acid rock drainage. Minerals Engineering, 24, 1277-1287.

Parbhakar-Fox, A., Fox, N., Ferguson, T., Hill, R. \& Maynard, B. (2018). Dissection of the NAG pH Test: Tracking Efficacy Through Examining Reaction Products. In: C. Wolkersdorfer, Sartz, L., Weber, A., Burgess, J., \& 
Tremblay, G. (Eds.), Mine Water - Risk to Opportunity (Vol II) (pp. 949-956). Pretoria, South Africa (Tshwane University of Technology).

Pelkonen, K., Alopeus, E., \& Penttilä, S. (1973). Outokumpu oy:N Hammaslahden kaivos. Vuoriteollisuus, 31(2), 90-96 (in Finnish).

Plumlee, G. S. (1999). The environmental geology of mineral deposits. In G. S. Plumlee, \& Logsdon, M. J. (Eds.), The Environmental Geochemistry of Ore Deposits. Part A: Processes, Techniques, and Health Issues (pp 71-116). Reviews in Economic Geology.

Price, W. A. (2003). Challenges posed by metal leaching and acid rock drainage, and approaches used to address them. In J. L. Jambor, Blowes, D. W., \& Ritchie, A. I. M. (Eds.), Environmental aspects of mine wastes. Mineralogical Association of Canada, Short Course Series, vol. 31, (pp. 15-30).

Price, W. A. (2009). Prediction Manual for Drainage Chemistry from Sulfidic Geologic Materials. Natural Resources Canada. MEND Report 1.20.1, 579 p.

Price, W. A., Morin, K., \& Hutt, N. (1997). Guidelines for prediction of acid rock drainage and metal leaching for mines in British Columbia: Part II. In Recommended procedures for static and kinetic tests. Fourth international conference on acid rock drainage. Vancouver, B.C. Canada, may 31 June 6, 1997. Proceedings, vol. I, 15-30.

Prosser, A. P. (1970). Influence of mineralogical factors on the rates of chemical reaction of minerals. In Mineral processing and extractive metallurgy" (IMM, London, 1970), vol. 3, (p. 59). 9th Commonwealth Mineral Metallurgy Congress (1969).

Ptacek, C. J., \& Blowes, D. W. (1992). Influence of siderite on the pore-water chemistry of inactive mine-tailings impoundment. In C. N. Alpers, \& Blowes, D. W. (Eds.), Environmental geochemistry of sulfide oxidation, (pp. 172-189). ACS symposium series 550 .

Räisänen, M. L., Kauppila, P. M., \& Myöhänen, T. (2010). Suitability of static tests for acid rock drainage assessment of mine waste rock. Bulletin of the Geological Society of Finland, 82, 101-111.

Rozalen, M., Ramos, M. E., Gervilla, F., Kerestedjian, T., Fiore, S., \& Huertas, F. J. (2014). Dissolution study of tremolite and anthophyllite: $\mathrm{pH}$ effect on the reaction kinetics. Applied Geochemistry, 49, 46-56.

Sanemasa, I., Yoshida, M., \& Ozawa, T. (1972). The dissolution of olivine in aqueous solutions of inorganic acids. Bulletin of the Chemical Society of Japan, 45, 1741-1746.

Santaguida, F., Luolavirta, K., Lappalainen, M., Ylinen, J., Voipio, T., \& Jones, S. (2015). The Kevitsa Ni-cu-PGE deposit in the Central Lapland greenstone belt in Finland. In W. Maier, R. Lahtinen, \& H. O'Brien (Eds.), Mineral Deposits of Finland. Elsevier ISBN: 978-0-12-410438-9.

Schweda, P., \& Kalinowski, B. (1994). Dissolution rates and alteration of muscovite, phlogopite and biotite at $\mathrm{pH} 1$ to 4 , room temperature. Edinburgh: Goldschmidt conference proceedings (pp. 817-818).

Seal, R. R. II, \& Hammarstrom, J. M. (2003). Geoenvironmental models of mineral deposits: Examples from massive sulfide and gold deposits. In J. L. Jambor, Blowes, D. W. \& Ritchie,
A. I. M. (Eds.), Environmental aspects of mine wastes, mineralogical Association of Canada, vol. 31, (pp. 11-50).

Singer, P. C., \& Stumm, W. (1970). Acid mine drainage-rate determining step. Science, 167, 1121-1123.

Smart, R., Skinner, W. M., Levay, G., Gerson, A. R., Thomas, J. E., Sobieraj, H., Schumann, R., Weisener, C. G., Weber, P. A., Miller, S. D., \& Stewart, W. A. (2002). ARD test handbook: Project P387A, A Prediction and Kinetic Control of Acid Mine Drainage. Melbourne: AMIRA international Ltd $42 \mathrm{p}$.

Sobek, A. A., Schuller, W. A., Freeman, J. R., \& Smith, R. M. (1978). Field and laboratory methods applicable to overburdens and Minesoils (p. 45268). Cincinnati: U.S. Environmental Protection Agency EPA-600/2-78-054, 47-50.

Sorjonen-Ward, P. (1993). An overview of structural evolution and lithic units within and intruding the late Archaean Hattu schist belt, Ilomantsi, eastern Finland. Geological Survey of Finland, Special Paper, 17, 9-102.

Sorjonen-Ward, P., \& Luukkonen, E. J. (2005). Archean rocks. In M. Lehtinen, P. A. Nurmi, \& O. T. Rämö (Eds.), Precambrian geology of Finland - Key to the evolution of the Fennoscandian shield (pp. 19-99). Amsterdam: Elsevier.

Stewart, W. A. (2005). Development of acid rock drainage prediction methodologies for coal mine wastes. PhD Dissertation, University of South Australia.

Stewart, W. A., Miller, S. D. \& Smart, R. (2006). Advances in acid rock drainage (ARD) characterization of mine wastes. In Proceedings of the 7th international conference in acid rock drainage, 2098-2119

Stumm, W., \& Morgan, J. J. (1996). Aquatic chemistry. New York: Wiley $1022 \mathrm{p}$.

Sverdrup, H. U. (1990). The kinetics of base cation release due to chemical weathering. Lund: Lund University Press.

Terry, B. (1983). The acid decomposition of silicate minerals. Part I. Reactivities and modes of dissolution of silicates. Hydrometallurgy, 10, 135-150.

Thomas, J. E., Jones, C. F., Skinner, W. M., \& Smart, R. S. C. (1998). The role of surface sulfur species in the inhibition of pyrrhotite dissolution in acid solution. Geochimica et Cosmochimica Acta, 65, 1-12.

Västi, K., Nikander, J., Kontoniemi, O., \& Eilu, P. (2012). F026 Laivakangas. In P. Eilu, T. Ahtola, O. Äikäs, T. Halkoaho, P. Heikura, H. Hulkki, M. Iljina, H. Juopperi, T. Karinen, N. Kärkkäinen, J. Konnunaho, A. Kontinen, O. Kontoniemi, E. Korkiakoski, M. Korsakova, T. Kuivasaari, M. Kyläkoski, H. Makkonen, T. Niiranen, J. Nikander, V. Nykänen, J.-A. Perdahl, E. Pohjolainen, J. Räsänen, P. Sorjonen-Ward, M. Tiainen, M. Tontti, A. Torppa, \& K. Västi (Eds.), Metallogenic areas in Finland (pp. 207-342). Finland, Special Paper 53: Geological Survey of Finland.

Verburg, R., Bezuidenhout, N., Chatwin, T., \& Ferguson, K. (2009). The global acid rock drainage guide (GARD guide). Mine Water Environment, 28, 305-310.

White III, W. W., Lapakko, K. A., \& Cox, R. L. (1999). Static-test methods most commonly used to predict acid mine drainage: Practical guidelines for use and interpretation. In G. S. Plumlee, \& Logsdon, M. (Eds.), The environmental geochemistry of mineral deposits. Part a: Processes, techniques, and health issues, vol. 6A (pp. 325-338). Reviews in Economic Geology. 